Supporting Information

for the

Article Entitled

\title{
Optical Purity as a Programmable Variable for Controlling Polyolefin Tacticity in Living Coordinative Chain Transfer Polymerization: Application to the Stereomodulated LCCTP of $\alpha, \omega$-Nonconjugated Dienes
}

\author{
Authored by \\ Mark A. Wallace, Charlotte M. Wentz, and Lawrence R. Sita* \\ Laboratory for Applied Catalyst Science and Technology \\ Department of Chemistry and Biochemistry \\ University of Maryland, College Park, Maryland 20742
}

Details for the polymerization and isolation of poly(methylene-1,3-cyclohexane) and poly(methylene-1,3-cyclopentane) materials. Supporting data for the characterization of polymeric materials including ${ }^{1} \mathrm{H}$ and ${ }^{13} \mathrm{C}\left\{{ }^{1} \mathrm{H}\right\}$ NMR Spectra, XRD traces, and DSC Traces. 


\section{Experimental Details}

\section{General Considerations}

All manipulations with air and moisture sensitive materials were carried out under $\mathrm{N}_{2}$ using standard Schlenk or glovebox techniques. Toluene (ReagentPlus, 99\%) was dried and deoxygenated by passage over activated alumina and activated copper catalyst (GetterMax 135) and collected under $\mathrm{N}_{2}$ prior to use. Chlorobenzene (ReagentPlus, $99 \%$ ) was dried over calcium hydride by refluxing at $130^{\circ} \mathrm{C}$ for three days and distilled under $\mathrm{N}_{2}$ prior to use. Chloroform- $d_{1}$ and tetrachloroethane- $d_{2}$ were purchased from Cambridge Isotopes and used as received. 1,5-Hexadiene and 1,6-heptadiene were purchased from $\mathrm{TCl}$ Chemicals, dried over $\mathrm{Na} / \mathrm{K}$ alloy and isolated by vacuum-transfer prior to use. $\left[\mathrm{PhNMe} \mathrm{H}_{2}\right]\left[\mathrm{B}\left(\mathrm{C}_{6} \mathrm{~F}_{5}\right)_{4}\right]$ was purchased from Boulder Scientific and used as received. Catalyst $\left(\boldsymbol{R}_{\mathrm{c}}, \boldsymbol{R}_{\mathrm{Hf}}\right)-\mathbf{1}$ and $\left(\boldsymbol{S}_{\mathrm{c}}, \boldsymbol{S}_{\mathrm{Hf}}\right)$-1 were synthesized according to published procedures. ${ }^{1}$

\section{Characterization}

Gel Permeation Chromatography (GPC) was used to obtain molecular weight (Mn and $\mathrm{Mw}$ ) and polydispersity index $(\boxminus)$ of polymers using Viscotek GPCMax equipped with 3 columns (Waters Styragel HR1, HR3 and HR4) in a column oven and differential refractometer maintained at $40{ }^{\circ} \mathrm{C}$. Tetrahydrofuran (HPLC Grade) was used as the eluent with a flow rate of $1 \mathrm{~mL} / \mathrm{min}$. Polystyrene standards (from Polymer Laboratories Inc. were used for calibration. For GPC sample preparation, $2 \mathrm{mg}$ of dry polymer sample were dissolved in $1 \mathrm{~mL}$ of THF (HPLC Grade).

Nuclear Magnetic Resonance (NMR) Spectroscopy for ${ }^{1} \mathrm{H}$ and ${ }^{13} \mathrm{C}\left\{{ }^{1} \mathrm{H}\right\}$ were carried out on a Brucker AVIII-HD 800 spectrometer fitted with a Cryo-QCl probe and a Bruker AV III HD NanoBay $400 \mathrm{MHz}$ spectrometer fitted with a BBFO probe. Chloroform- $d_{1}$ was used as the solvent for low MW polymer samples. Tetrachloroethane- $d_{2}$ was used for high MW polymer samples with spectra collected at $110^{\circ} \mathrm{C}$ after equilibrating the sample for twenty minutes. Spectra were referenced using residual ${ }^{1} \mathrm{H}$ and ${ }^{13} \mathrm{C}\left\{{ }^{1} \mathrm{H}\right\}$ chemical shifts of the deuterated solvents. ${ }^{13} \mathrm{C}\left\{{ }^{1} \mathrm{H}\right\}$ NMR assignments for the four limiting microstructures of poly(methylene-1,3-cyclohexane) (Figure S1) and poly(methylene-1,3-cyclopentane) (Figure S2) were made according to previously reported assignments. ${ }^{1-4}$ 


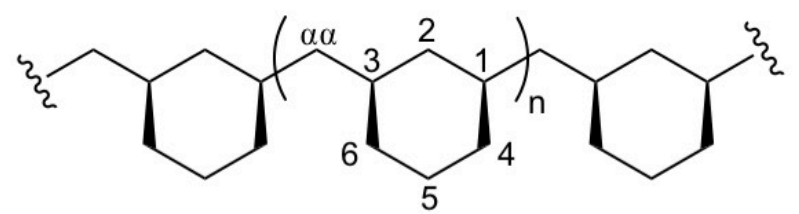

1,3-cis-isotactic (1,3-c-iso)

4,6-cis-isotactic (4,6-c-iso)

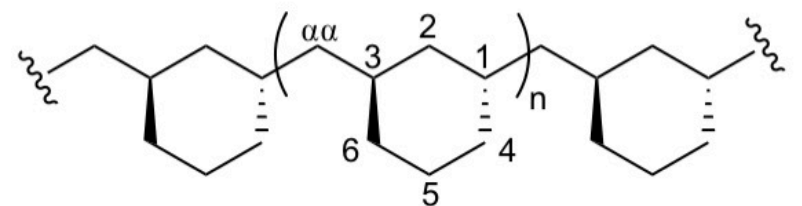

1,3-trans-isotactic $(1,3-t)$

4,6-trans-isotactic $(4,6-t)$

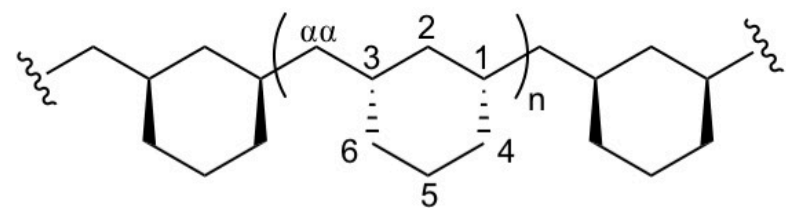

1,3-cis-syndiotactic (1,3-c-syn) 4,6-cis-syndiotactic (4,6-c-syn)

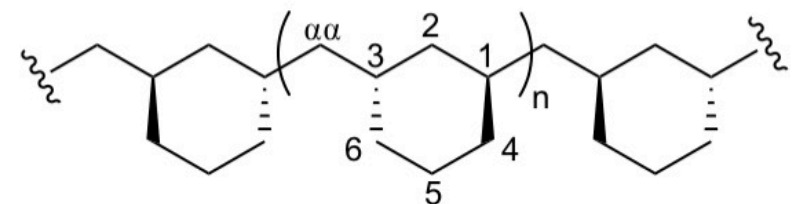

1,3-trans-syndiotactic $(1,3-t)$ 4,6-trans-syndiotactic (4,6- $t)$

Figure S1. Limiting stereochemical microstructures of poly(methylene-1,3-cyclohexane).<smiles>[Z2]CC1CC[C@@](C)(C[C@H]2CC[C@H](C(C)(C)C3CC[C@H]([Z2])C3)C2)C1</smiles>

cis, isotactic

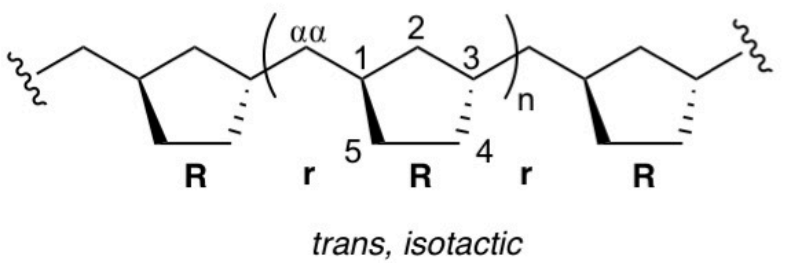<smiles>[Z2]CC1CC[C@@](C)(CC(C)(C)CC(C)C(C)(C)C2CC[C@H]([Z2])C2)C1</smiles>

cis, syndiotactic

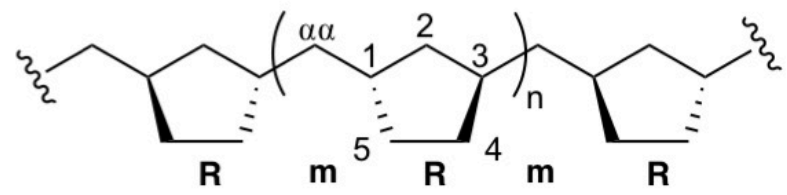

trans, syndiotactic

Figure S2. Limiting stereochemical microstructures of poly(methylene-1,3-cyclohexane). 
Powder X-ray Diffraction (XRD) of samples was carried out with a D8 Advanced diffractometer equipped with a LynxEye detector under ambient conditions. On a scatter less sample holder the sample was compacted in a circle with a diameter of $2 \mathrm{~mm}$ for measurement. X-rays from a $\mathrm{Cu} \mathrm{K} \alpha$ radiation source with a wavelength of $1.5418 \AA$ was used, and the $\theta$-angle starting at $5^{\circ}$ ending at $60^{\circ}$ with a $0.05^{\circ}$ step. The data profiles were processed using Advanced TOPAS.

Differential Scanning Calorimetry (DSC) was performed using a TA instruments DSC Q1000 system. Samples were run in sealed T-Zero pans with an empty pan as reference. A heat $/ \mathrm{cool} /$ heat $/ \mathrm{cool}$ temperature program was used at a heating rate of $10{ }^{\circ} \mathrm{C} / \mathrm{min}$ and cooling rate of $5^{\circ} \mathrm{C} / \mathrm{min}$ from $-5^{\circ} \mathrm{C}-220^{\circ} \mathrm{C}$ for all $\mathrm{PMCH}$ materials. The $\mathrm{Tm}$ of run 1 was collected using a heat $/ \mathrm{cool} / \mathrm{heat} / \mathrm{cool}$ cycle at a heating rate of $10{ }^{\circ} \mathrm{C} / \mathrm{min}$ and cooling rate of $10{ }^{\circ} \mathrm{C} / \mathrm{min}-70^{\circ} \mathrm{C}-250{ }^{\circ} \mathrm{C}$. A heat/cool/heat temperature program was used at a heating rate of $10^{\circ} \mathrm{C} / \mathrm{min}$ and cooling rate of $10^{\circ} \mathrm{C} / \mathrm{min}$ from $-70^{\circ} \mathrm{C}-250^{\circ} \mathrm{C}$ for all PMCP materials. The initial mass of the sample was $6-12 \mathrm{mg}$. Phase transition temperatures are reported for the second heat/cool cycle is

\section{Polymerization Procedures}

LCP of 1,6-heptadiene and 1,5-hexadiene. To a $25 \mathrm{~mL}$ round bottom with a $7 \mathrm{~mL}$ chlorobenzene solution of $38.0 \mathrm{mg}(0.048 \mathrm{mmols})$ of $\left[\mathrm{PhNMe}{ }_{2} \mathrm{H}\right]\left[\mathrm{B}\left(\mathrm{C}_{6} \mathrm{~F}_{5}\right)_{4}\right]$ chilled to $-5^{\circ} \mathrm{C}$ was added $24.6 \mathrm{mg}(0.044 \mathrm{mmols})$ of $\left(\boldsymbol{R}_{\mathrm{c}}, \boldsymbol{R}_{H f}\right)-1 \mathrm{in} 1 \mathrm{~mL}$ of chlorobenzene prechilled to $-5{ }^{\circ} \mathrm{C}$. The solution was stirred and allowed to equilibrate for 10 minutes, turning a paleyellow color. To the pale-yellow solution, $0.352 \mathrm{~g}$ (3.66 mmols) of 1,6-heptadiene prechilled to $-5^{\circ} \mathrm{C}$ was added all at once. The reaction mixture was stirred for 20 hours at $-5{ }^{\circ} \mathrm{C}$ before precipitating from $500 \mathrm{~mL}$ acidic methanol ( $10 \%$ concentrated $\mathrm{HCL}$ ). The polymer material was collected via filtration and washed $2 x$ with methanol. The polymer was dried overnight before characterization.

General Procedure for the LCCTP of 1,6-heptadiene and 1,5-hexadiene. To a $25 \mathrm{~mL}$ round bottom with a $7 \mathrm{~mL}$ toluene solution of $38.0 \mathrm{mg}(0.048 \mathrm{mmols})$ of $\left[\mathrm{PhNMe} \mathrm{H}^{\mathrm{H}}\right]\left[\mathrm{B}\left(\mathrm{C}_{6} \mathrm{~F}_{5}\right)_{4}\right]$ chilled to $-5^{\circ} \mathrm{C}$ was added $24.6 \mathrm{mg}(0.044 \mathrm{mmols})$ of $\left(\boldsymbol{R}_{\mathbf{c}}, \boldsymbol{R}_{\boldsymbol{H}}\right)-\mathbf{1}$ in $1 \mathrm{~mL}$ of chlorobenzene prechilled to $-5{ }^{\circ} \mathrm{C}$. The solution was stirred and allowed to equilibrate for 10 minutes, turning a pale-yellow color. $55.0 \mathrm{mg}(0.440 \mathrm{mmol})$ of ZnEt2 prechilled to $-5^{\circ} \mathrm{C}$ was added to the solution followed by $1.76 \mathrm{~g}(18.3 \mathrm{mmols})$ of $1,6-$ heptadiene, prechilled to $-5^{\circ} \mathrm{C}$, added all at once. The reaction mixture was stirred for 24 hours at $-5^{\circ} \mathrm{C}$ before quenching with $2.0 \mathrm{~mL}$ of acidic methanol $(10 \%$ concentrated $\mathrm{HCl})$. Solvent was removed in vacuo and residue was collected in hexanes and passed through a silica plug. Solvent was removed in vacuo and the isolated polymer was dried overnight before GPC and NMR analysis. Details on the amount of the reagents, polymerization time, and polymer characterization are provided in Table 1. 


\section{Supporting NMR Data}

A<smiles>C=CCCC(CC(C)CC1CCC(C)C1)CC1CCC(CC2CCC(C)C2)C1</smiles>
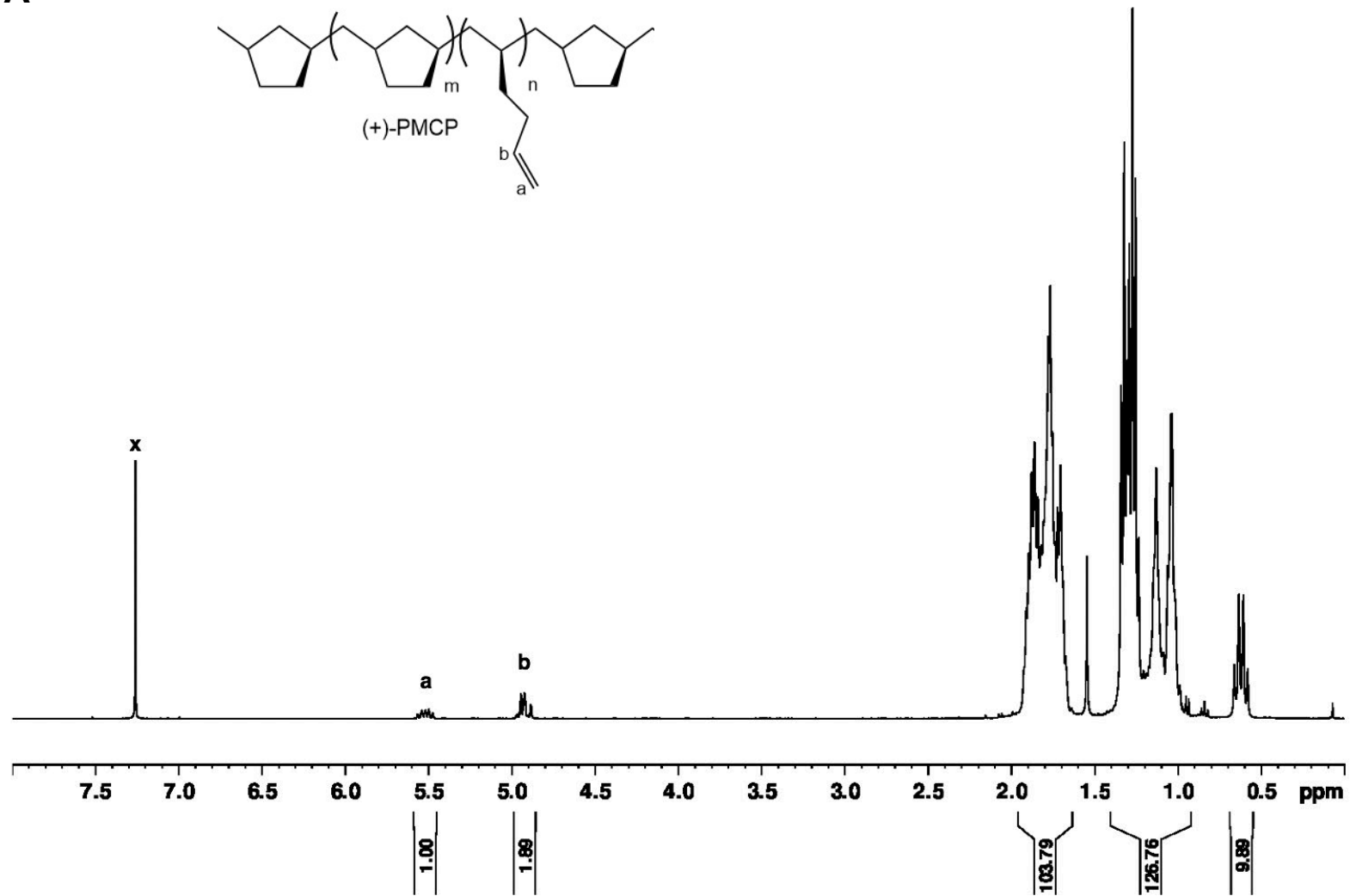

B

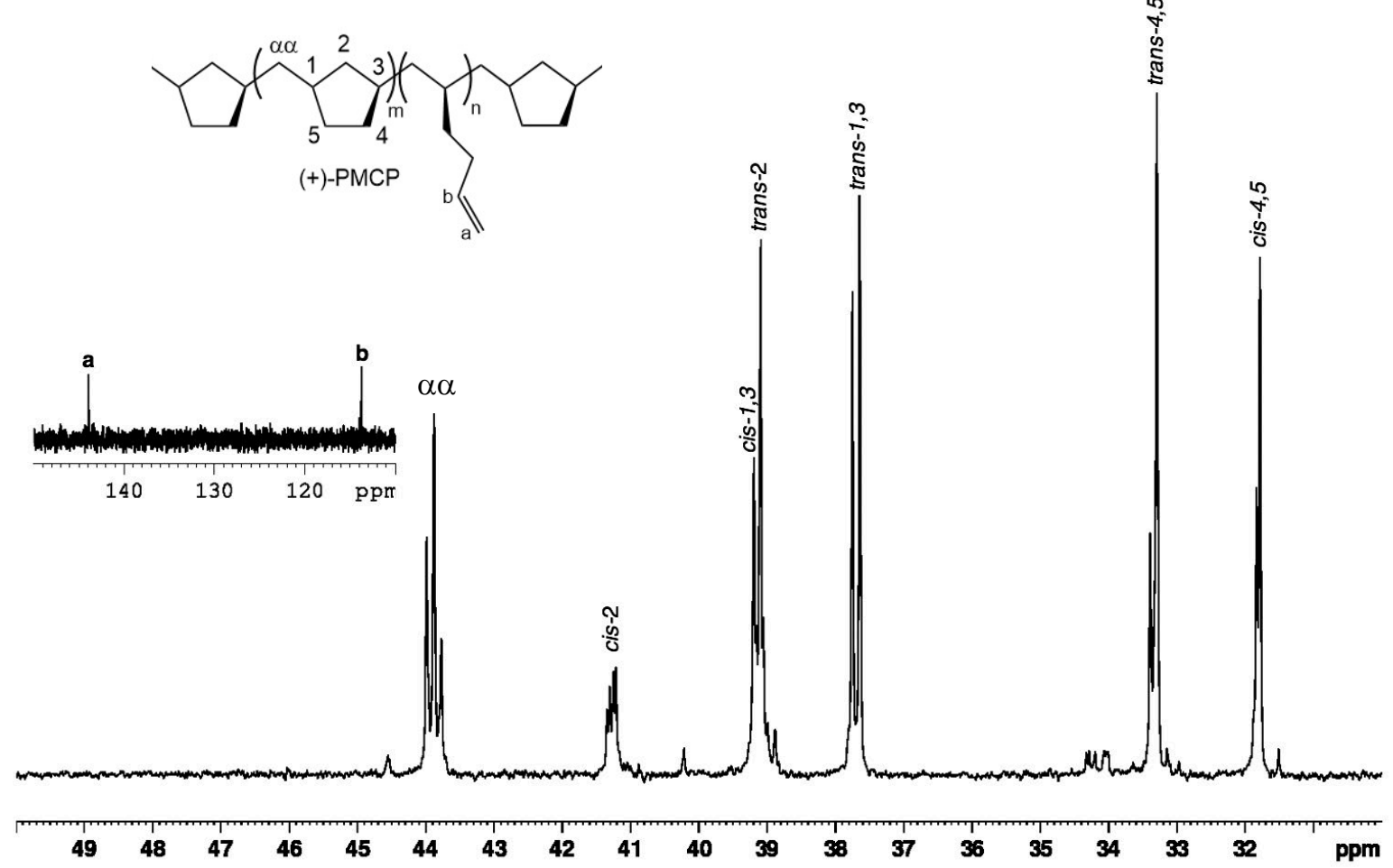

Figure S3. (A) ${ }^{1} \mathrm{H}$ NMR $\left(400 \mathrm{MHz}, \mathrm{CDCl}_{3}, 25^{\circ} \mathrm{C}\right)$ spectrum of $\mathrm{PMCP}$ from run 1. (B) Partial ${ }^{13} \mathrm{C}$ NMR $\left(100 \mathrm{MHz}, \mathrm{CDCl}_{3}, 25^{\circ} \mathrm{C}\right.$ ) spectrum of $\mathrm{PMCP}$ from run $\mathbf{1} ; \mathbf{X}$ denotes $\mathrm{CDCl}_{3}$ solvent 
A
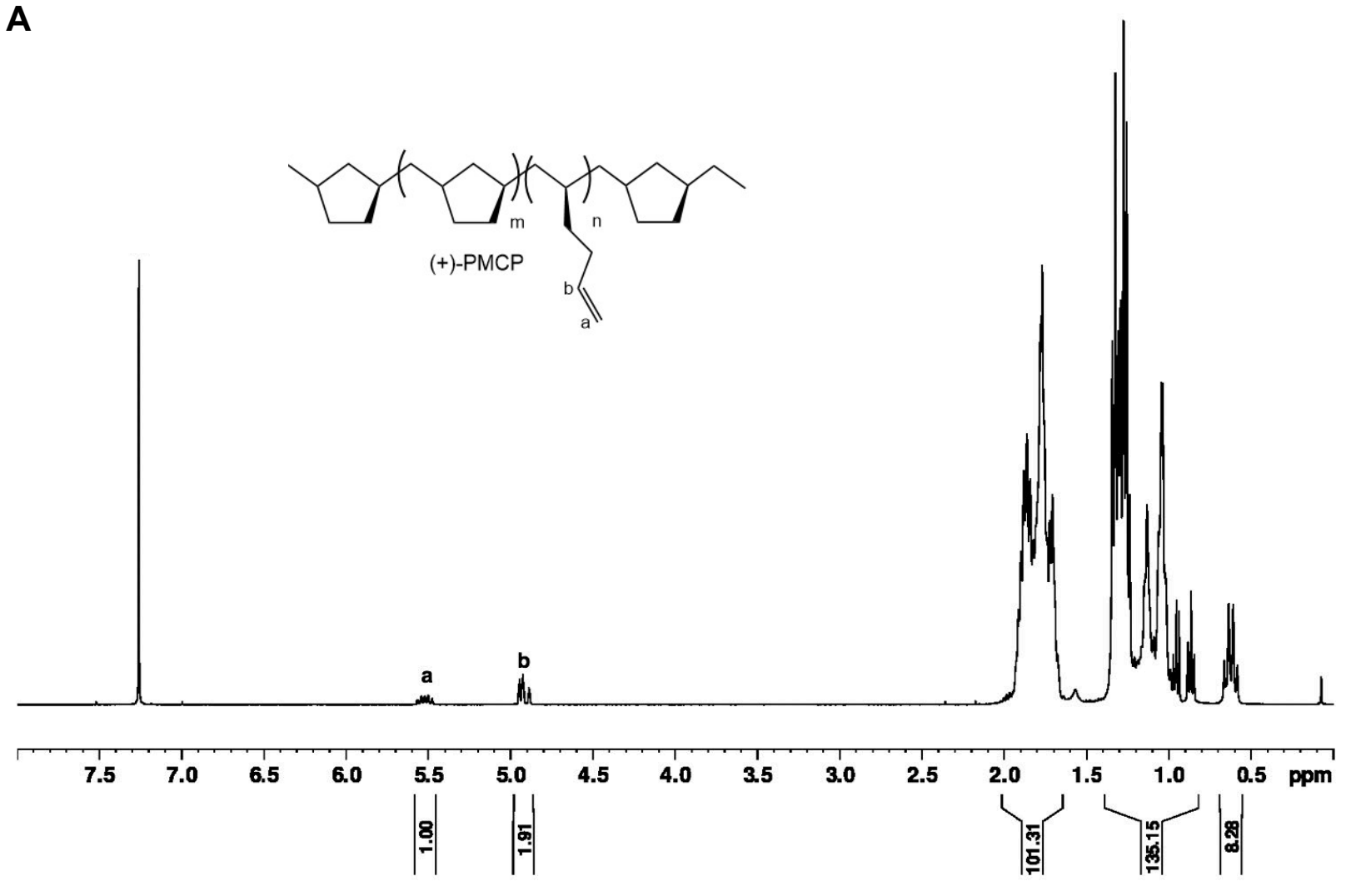

B

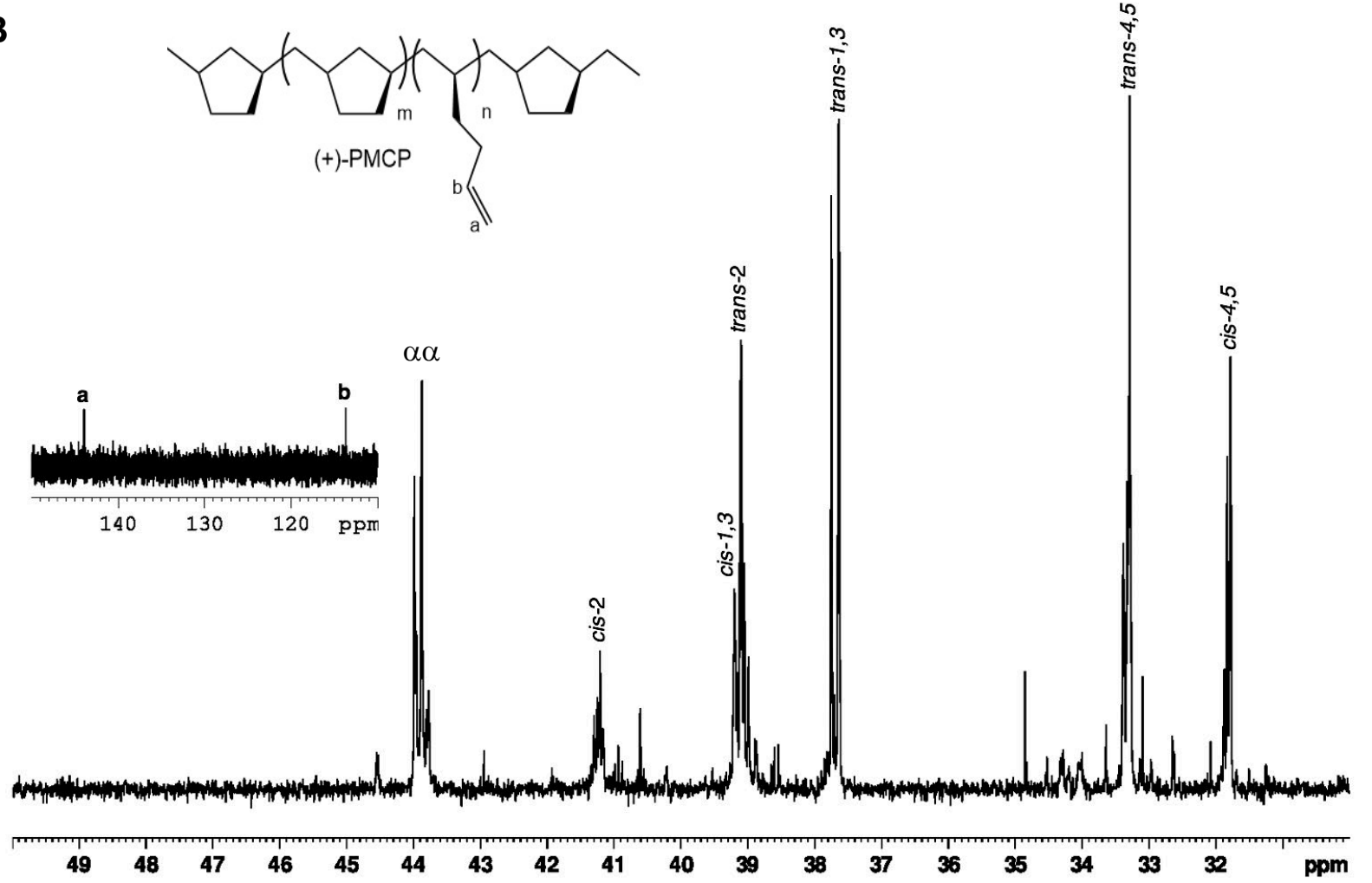

Figure S4. (A) ${ }^{1} \mathrm{H}$ NMR $\left(400 \mathrm{MHz}, \mathrm{CDCl}_{3}, 25^{\circ} \mathrm{C}\right)$ spectrum of $\mathrm{PMCP}$ from run 2. (B) Partial ${ }^{13} \mathrm{C}$ NMR $\left(100 \mathrm{MHz}, \mathrm{CDCl}_{3}, 25^{\circ} \mathrm{C}\right)$ spectrum of PMCP from run $2 ; \mathbf{X}$ denotes $\mathrm{CDCl}_{3}$ solvent. 
A

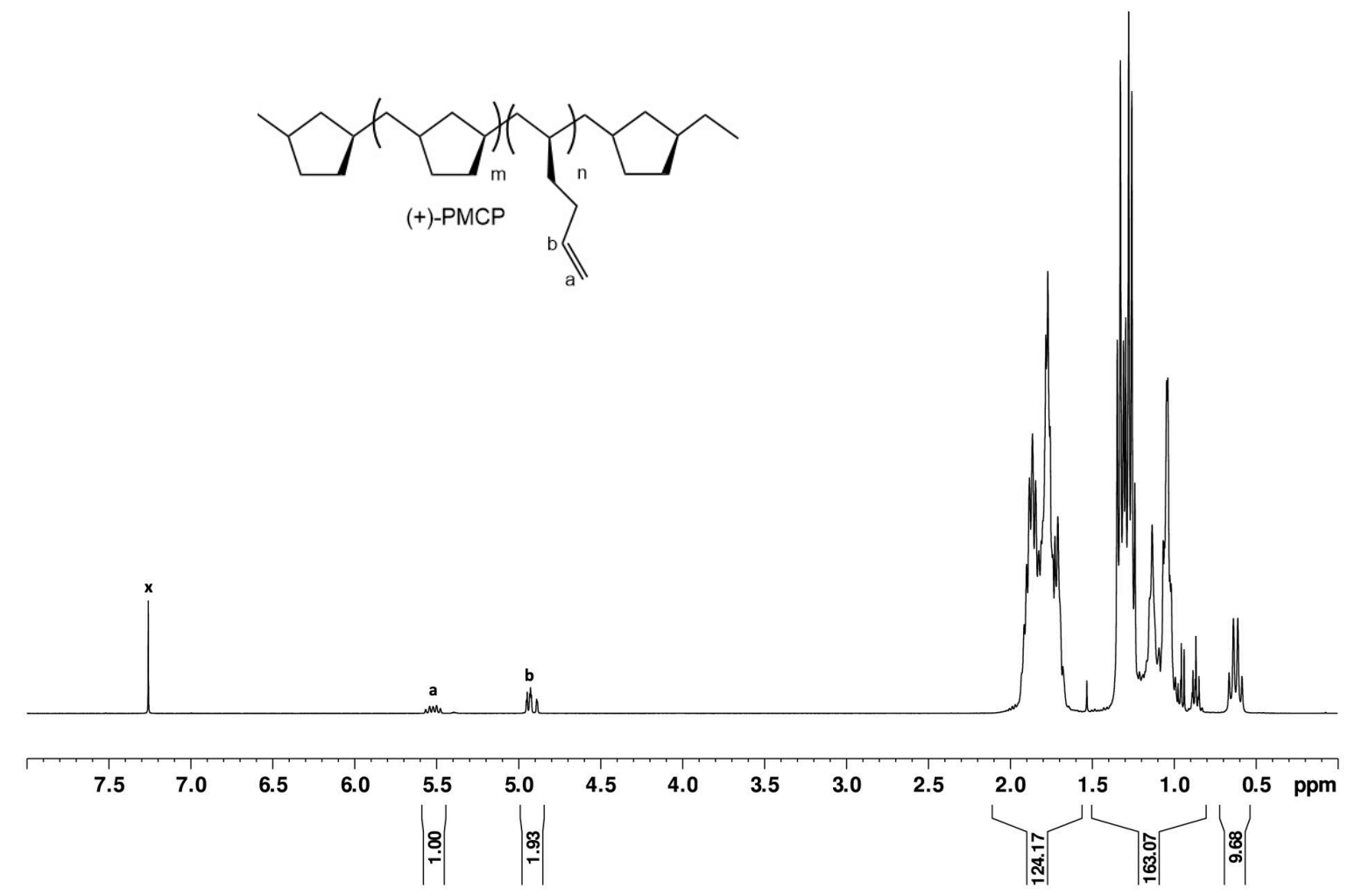

B

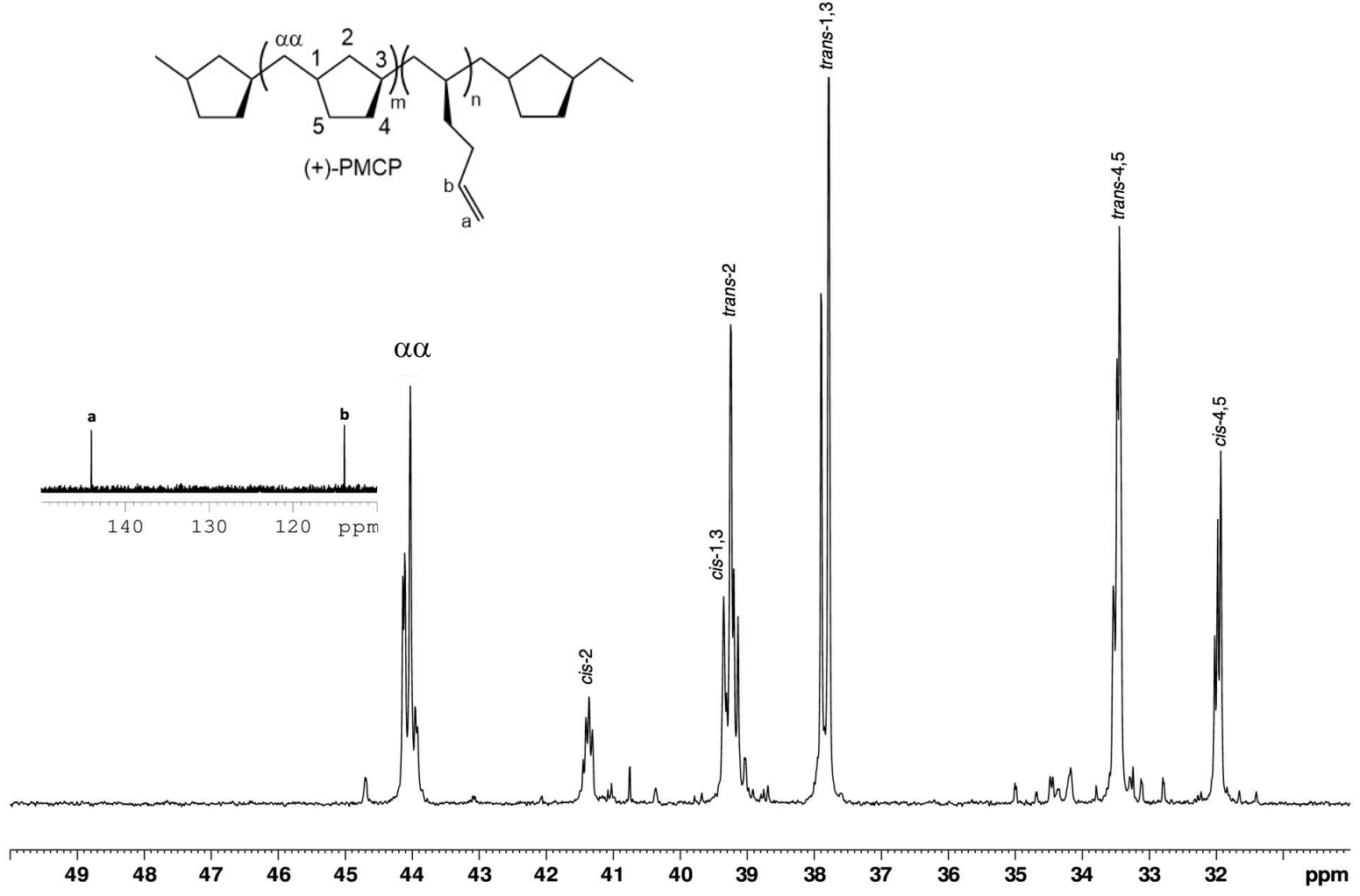

Figure S5. (A) ${ }^{1} \mathrm{H}$ NMR $\left(400 \mathrm{MHz}, \mathrm{CDCl}_{3}, 25{ }^{\circ} \mathrm{C}\right)$ spectrum of PMCP from run 3. (B) Partial ${ }^{13} \mathrm{C} \mathrm{NMR}$ $\left(100 \mathrm{MHz}, \mathrm{CDCl}_{3}, 25^{\circ} \mathrm{C}\right.$ ) spectrum of $\mathrm{PMCP}$ from run $3 ; \mathbf{X}$ denotes $\mathrm{CDCl}_{3}$ solvent. 
A
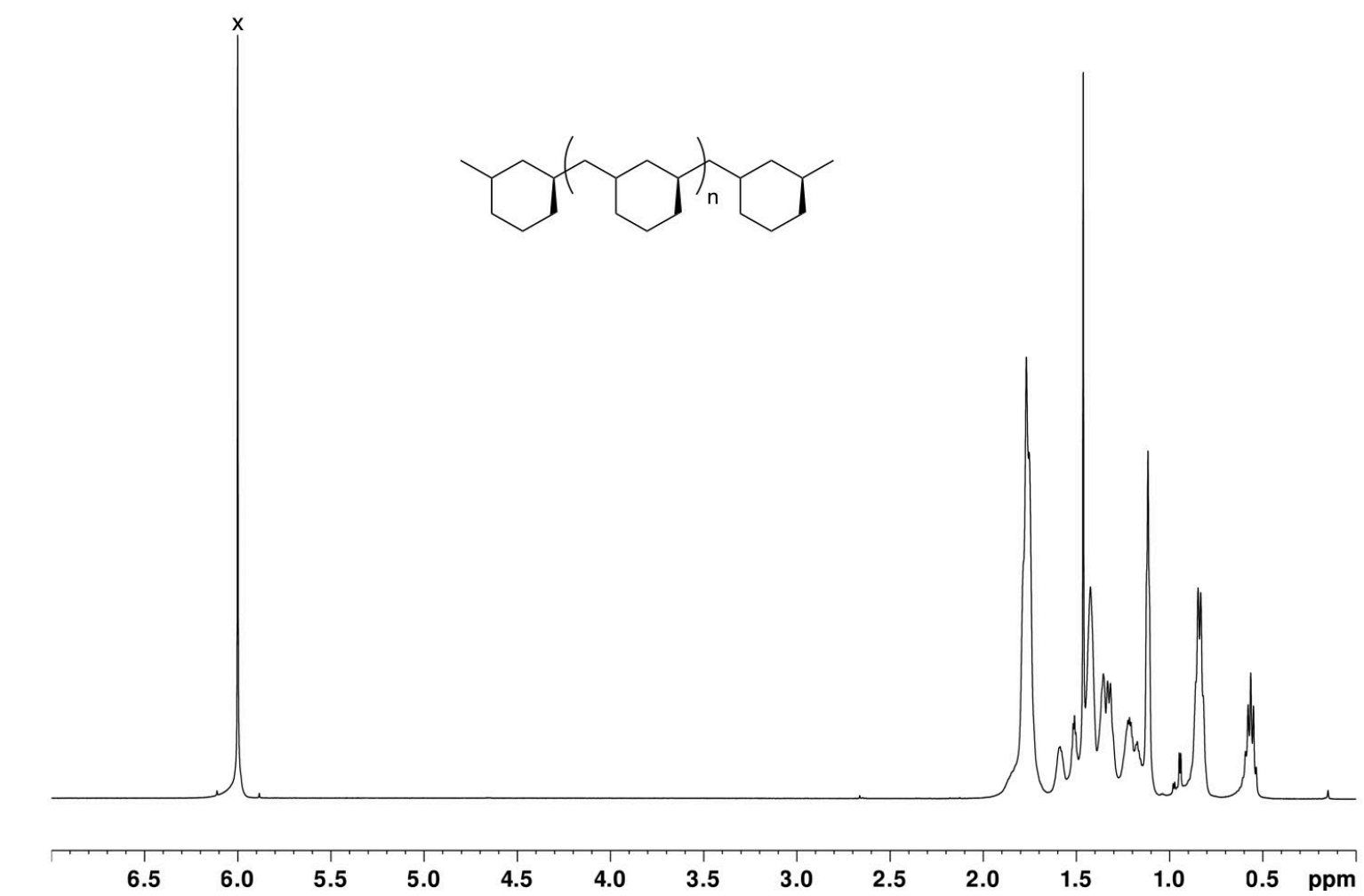

B

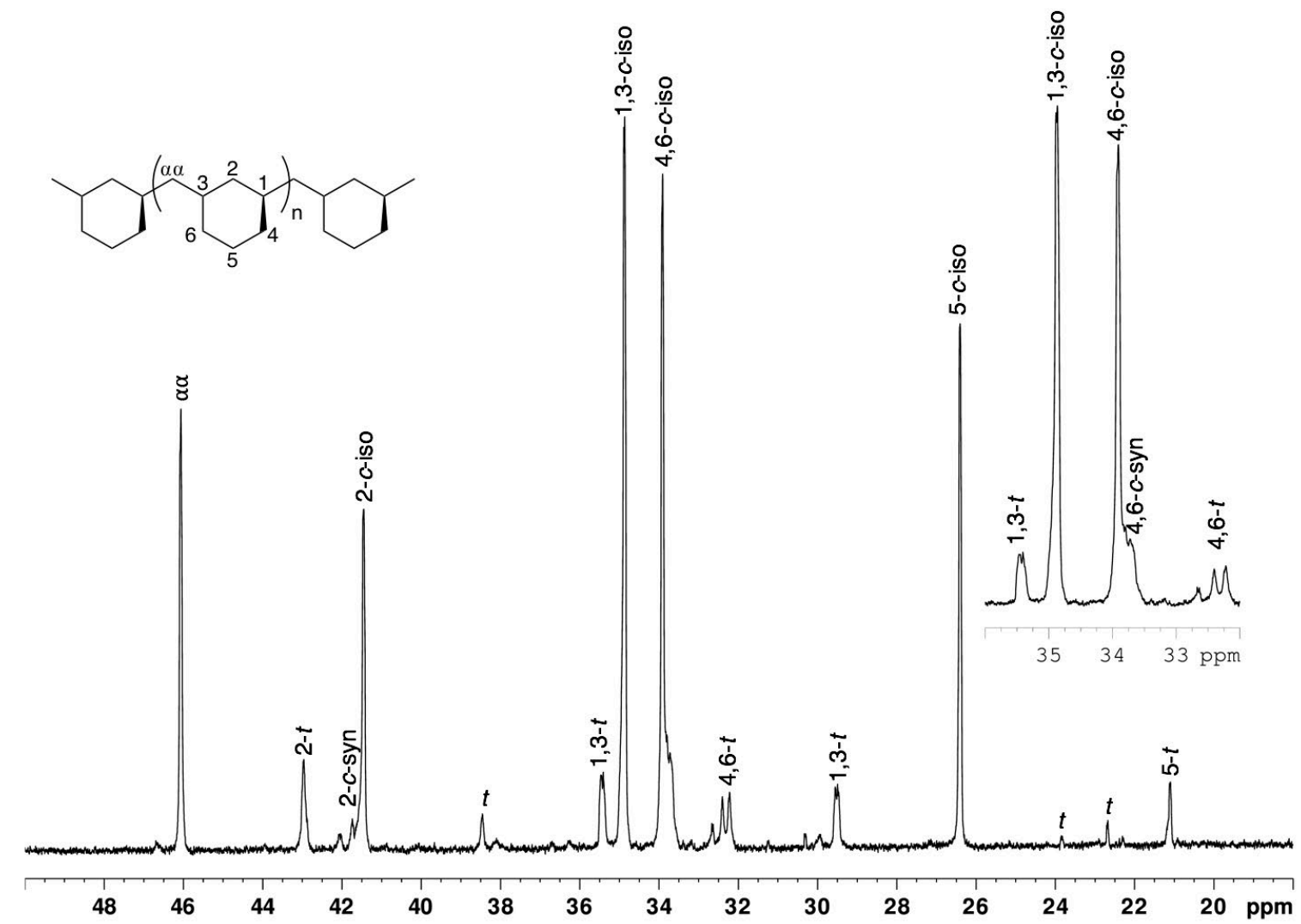

Figure S6. A) ${ }^{1} \mathrm{H}$ NMR $\left(800 \mathrm{MHz}, \mathrm{TCIE}-\mathrm{d}_{2}, 110^{\circ} \mathrm{C}\right)$ of $\mathrm{PMCH}$ from run 4. B) Partial ${ }^{13} \mathrm{C}\left\{{ }^{1} \mathrm{H}\right\} \mathrm{NMR}(200$ $\mathrm{MHz}, \mathrm{TCIE}-d_{2}, 110^{\circ} \mathrm{C}$ ) of $\mathrm{PMCH}$ from run 4 ; X denotes solvent peak. 
A<smiles>CCC1CCCC(C(C)(C)C2CCCC(CC3(C)CCCC(C)C3)C2)C1</smiles>

B
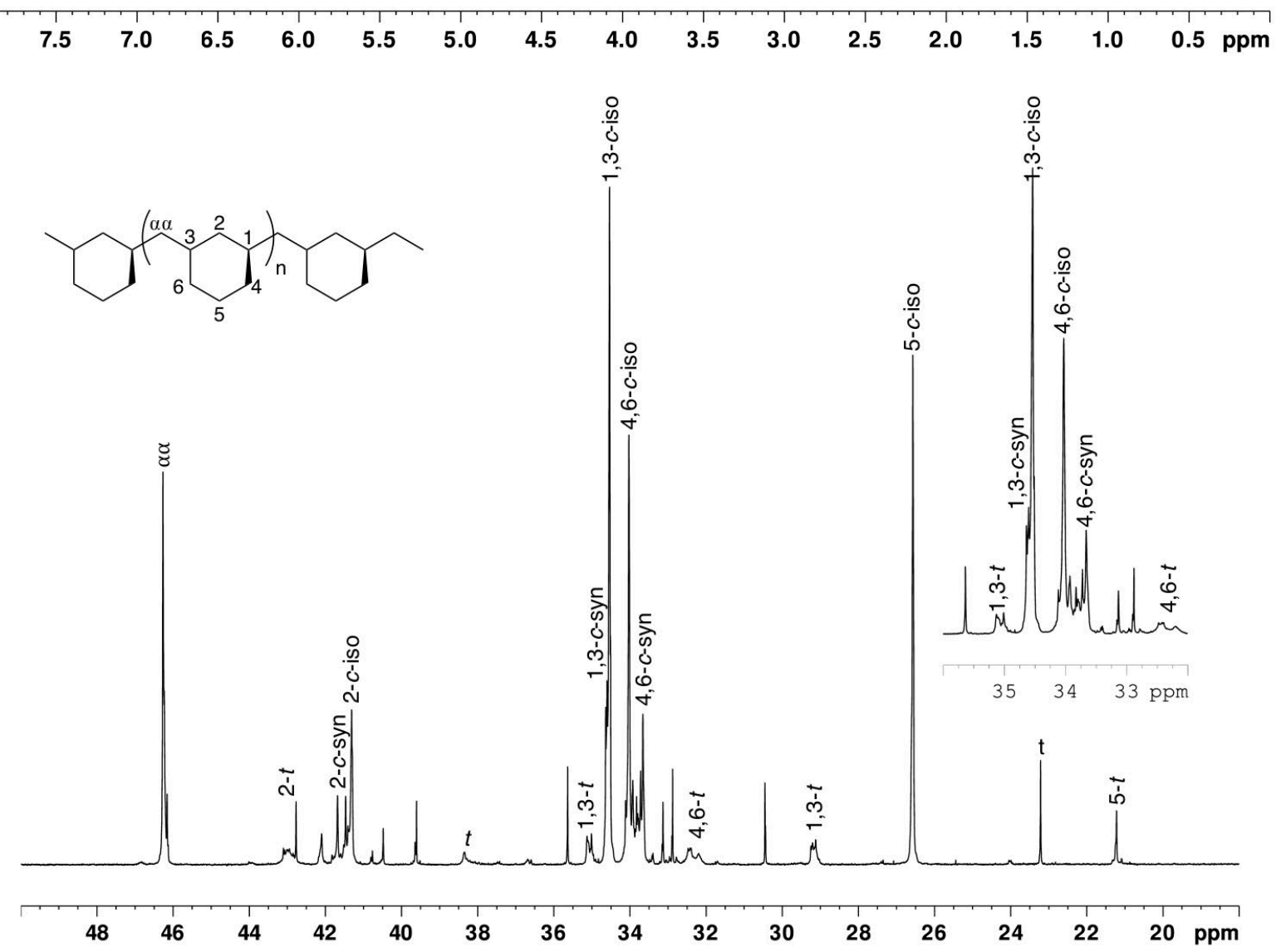

Figure S7. A) ${ }^{1} \mathrm{H}$ NMR $\left(800 \mathrm{MHz}, \mathrm{CDCl}{ }_{3}, 25^{\circ} \mathrm{C}\right)$ of $\mathrm{PMCH}$ from run 5. B) Partial ${ }^{13} \mathrm{C}\left\{{ }^{1} \mathrm{H}\right\} \mathrm{NMR}(200$ $\mathrm{MHz}, \mathrm{CDCl}_{3}, 25^{\circ} \mathrm{C}$ ) of $\mathrm{PMCH}$ from run 5 ; $\mathrm{X}$ denotes solvent peak. 
A
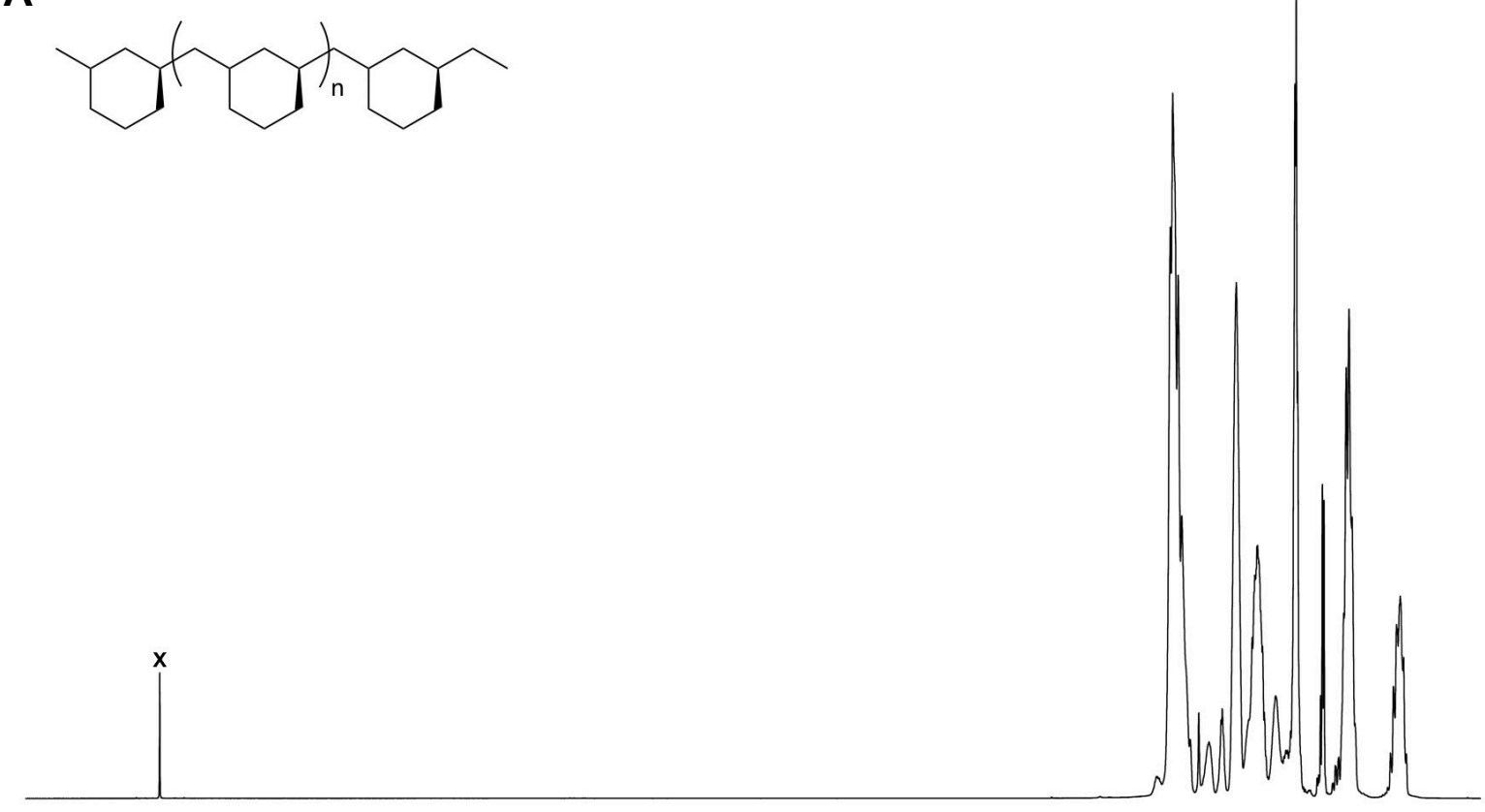

\begin{tabular}{llllllllllllllllll}
\hline 7.5 & 7.0 & 6.5 & 6.0 & 5.5 & 5.0 & 4.5 & 4.0 & 3.5 & 3.0 & 2.5 & 2.0 & 1.5 & 1.0 & 0.5 & ppm
\end{tabular}

B<smiles>CCC1CCCC(C(C)(C)C2CCCC(CC3(C)CCCC(C)C3)C2)C1</smiles> 
A<smiles>CCC1CCCC(C(C)C2CCCC(CC3(C)CCCC(C)C3)C2)C1</smiles>
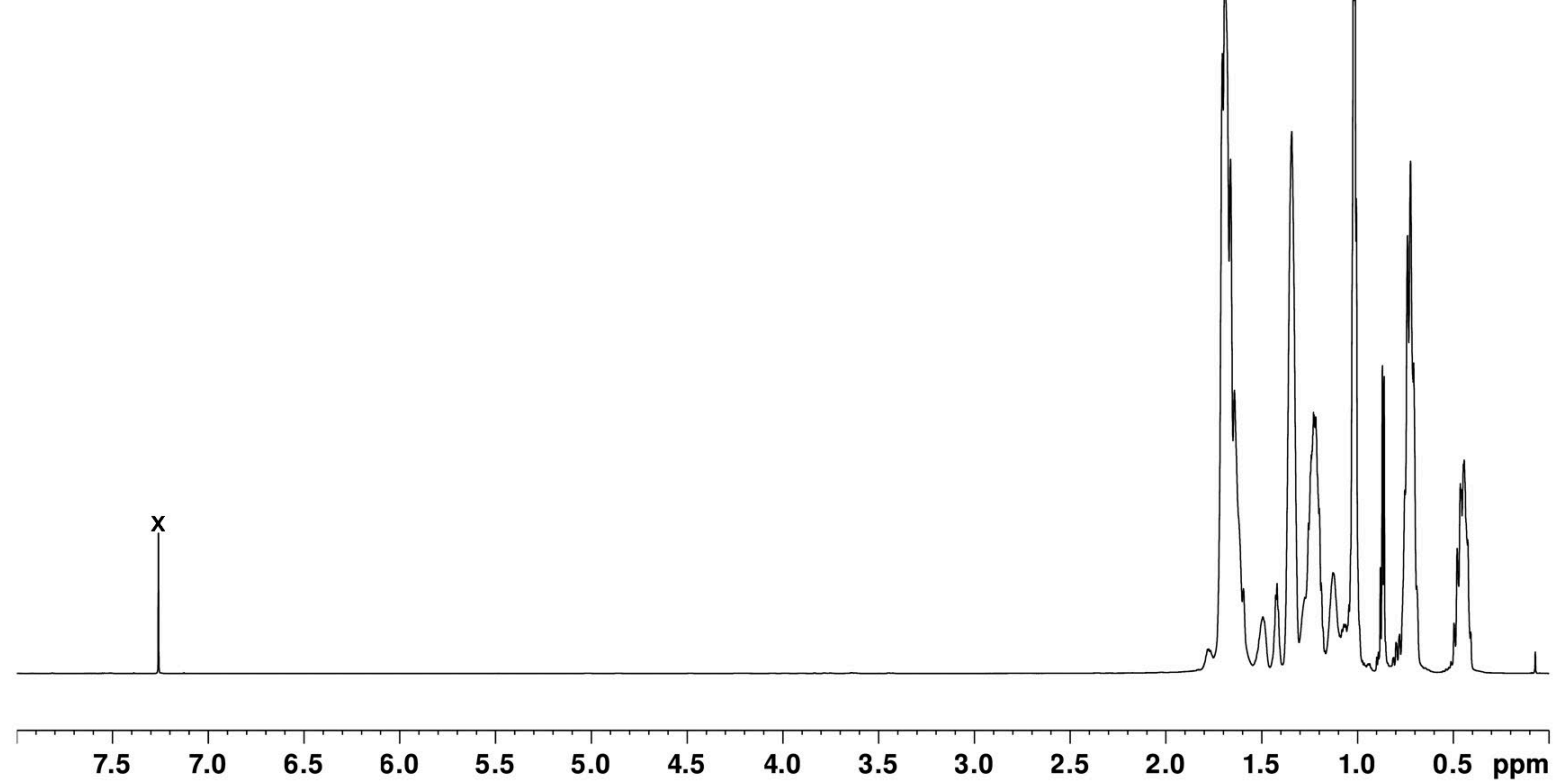

B<smiles>CCC1CCCC(C(C)(C)C2CCCC(CC3(C)CCCC(C)C3)C2)C1</smiles>
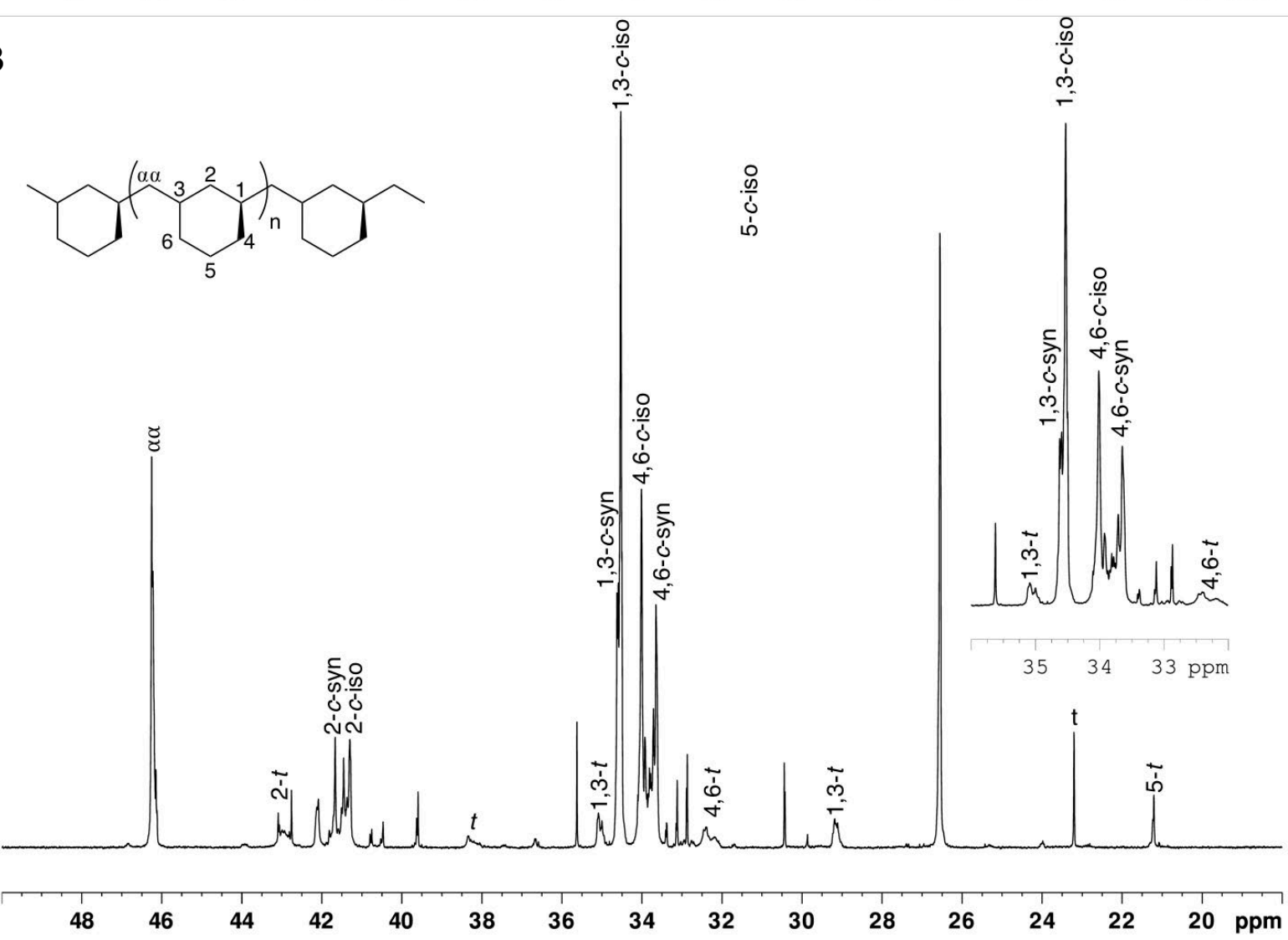

Figure S9. A) ${ }^{1} \mathrm{H}$ NMR (800 MHz, $\left.\mathrm{CDCl} 3,25{ }^{\circ} \mathrm{C}\right)$ of $\mathrm{PMCH}$ from run 7. B) Partial ${ }^{13} \mathrm{C}\left\{{ }^{1} \mathrm{H}\right\} \mathrm{NMR}(200$ $\mathrm{MHz}, \mathrm{CDCl}_{3}, 25^{\circ} \mathrm{C}$ ) of $\mathrm{PMCH}$ from run $7 ; \mathrm{X}$ denotes solvent peak. 
A<smiles>C[Te][Te]=[Te]</smiles>
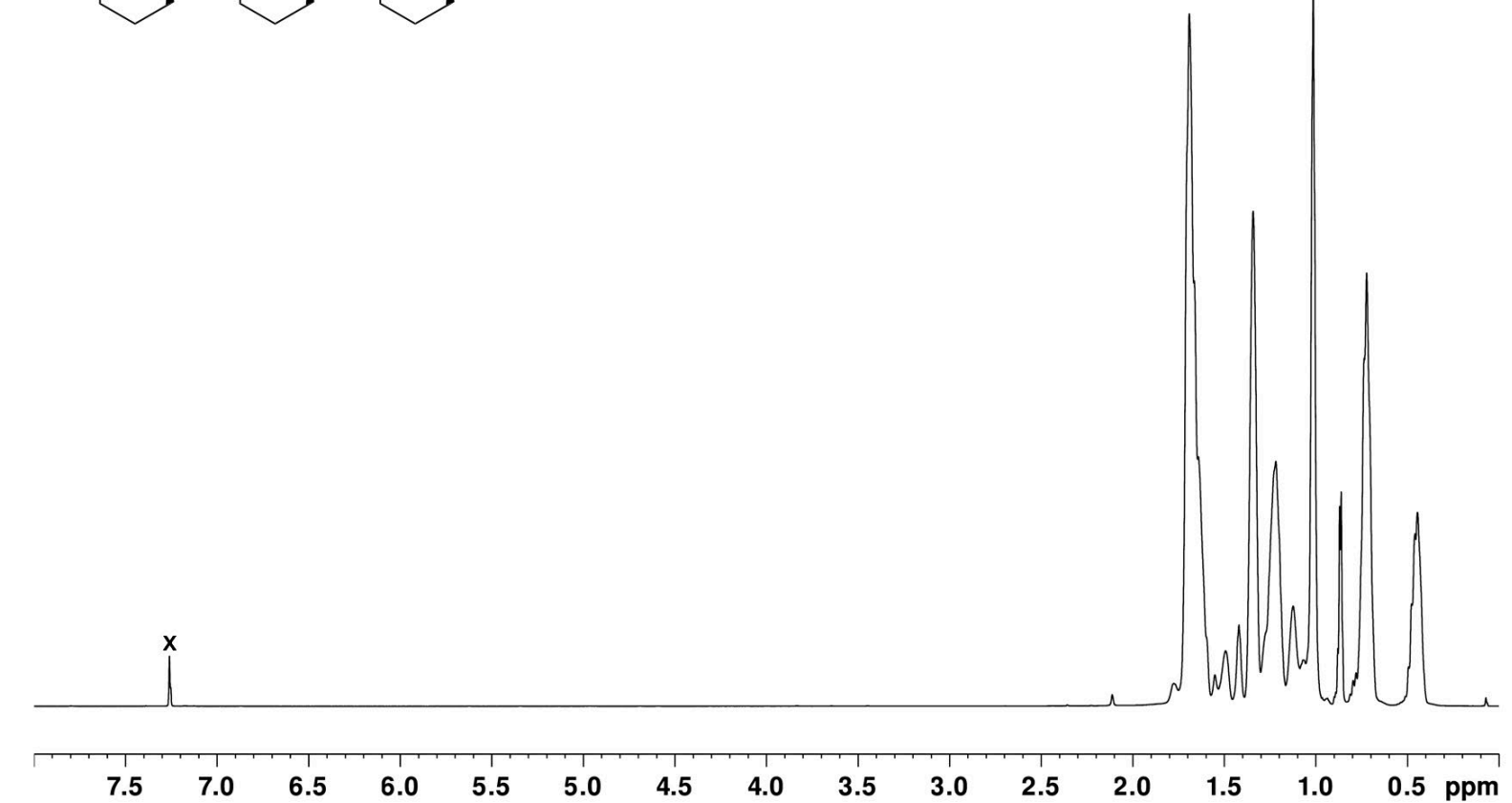

B<smiles>CCC1CCCC(C(C)C2CCCCC2C)C1</smiles>

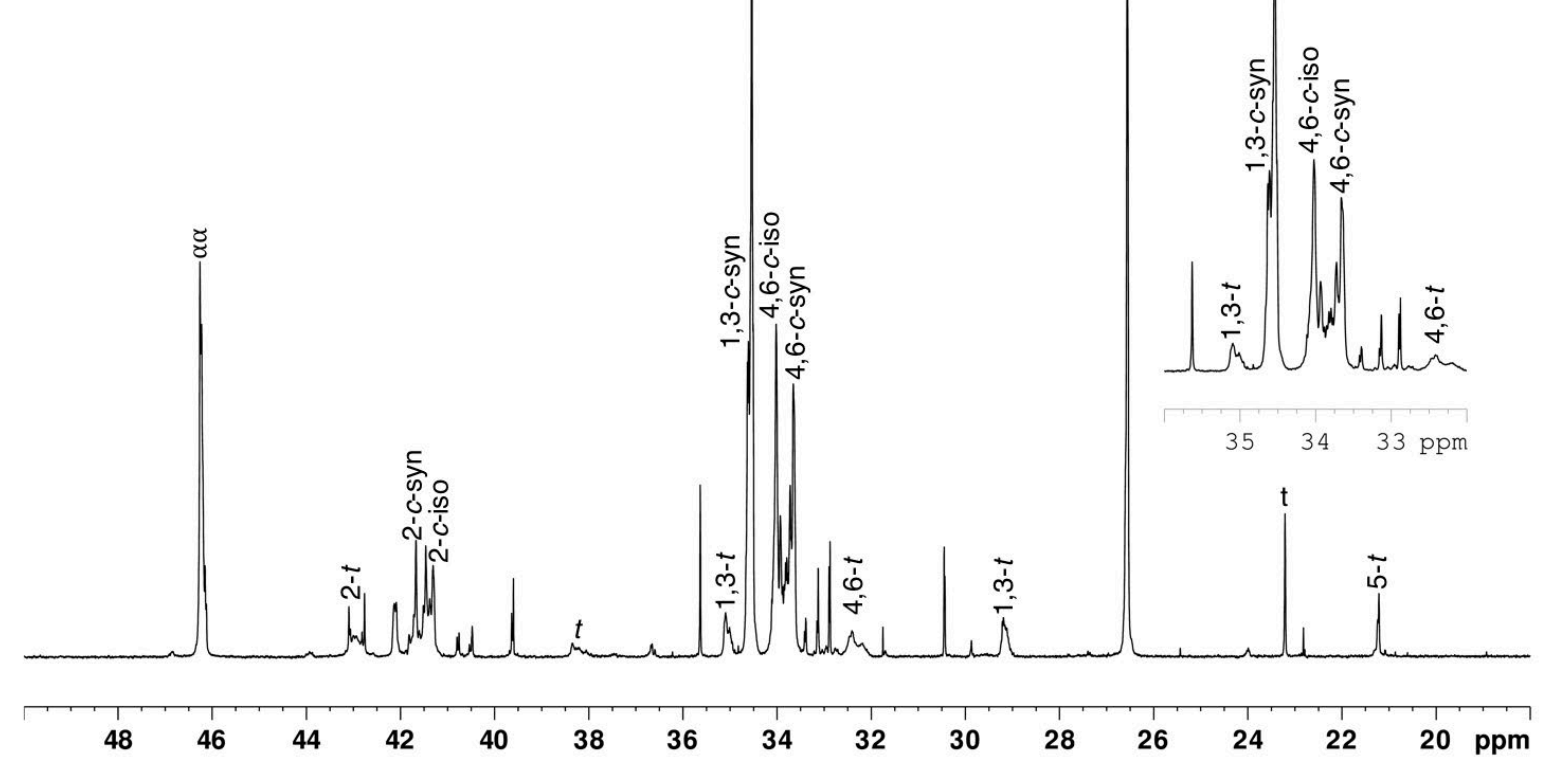

Figure S10. A) ${ }^{1} \mathrm{H}$ NMR $\left(800 \mathrm{MHz}, \mathrm{CDCl}_{3}, 25^{\circ} \mathrm{C}\right)$ of $\mathrm{PMCH}$ from run 8. B) Partial ${ }^{13} \mathrm{C}\left\{{ }^{1} \mathrm{H}\right\}$ NMR (200 $\mathrm{MHz}, \mathrm{CDCl}_{3}, 25^{\circ} \mathrm{C}$ ) of $\mathrm{PMCH}$ from run 8; $\mathrm{X}$ denotes solvent peak. 
A<smiles>CCC1CCCC(C(C)C2CCCC(CC3(C)CCCC(C)C3)C2)C1</smiles>
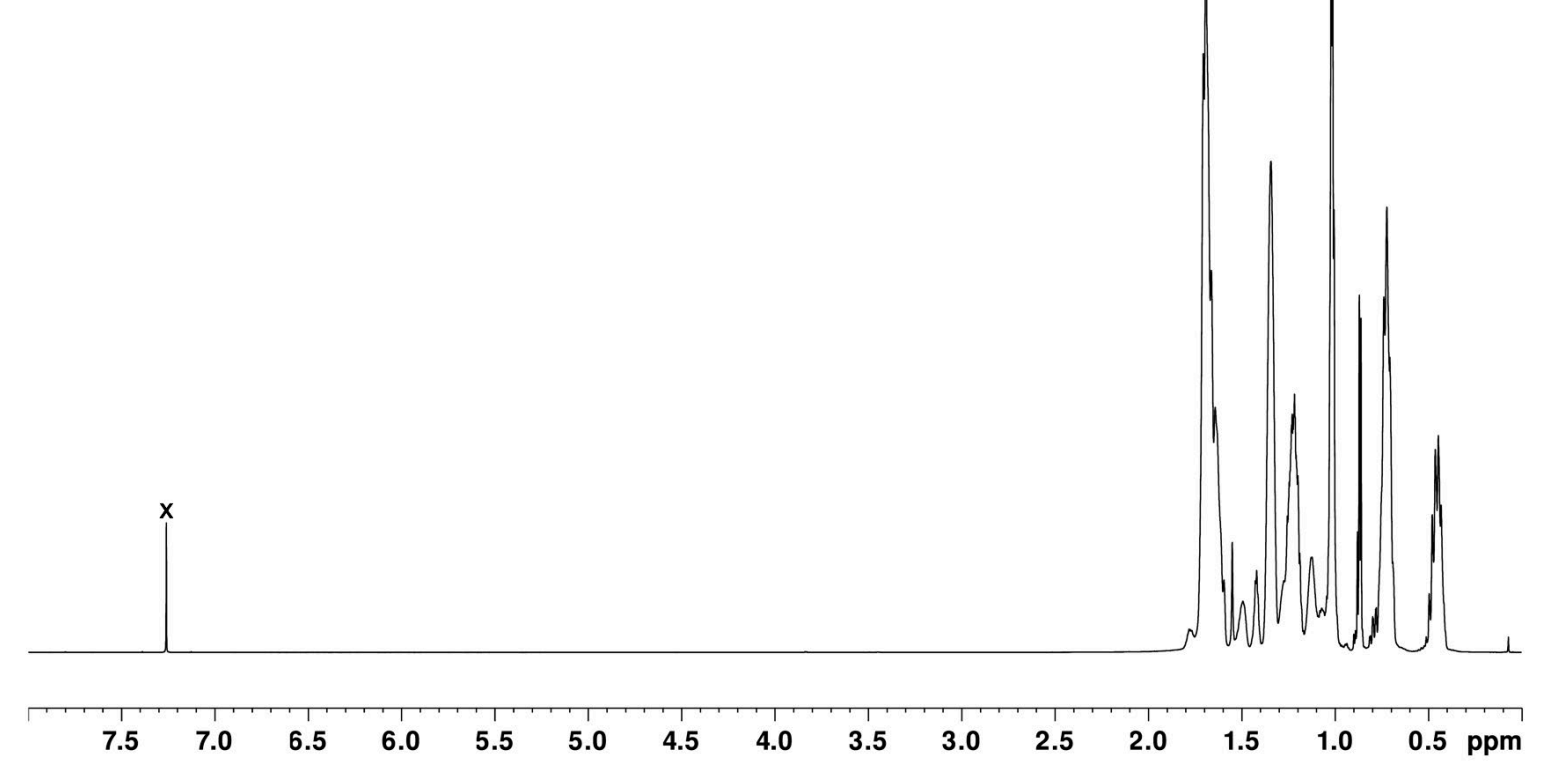

B<smiles>CCC1CCCC(C(C)C(C)C2CCCC(CC3(C)CCCC(C)C3)C2)C1</smiles>

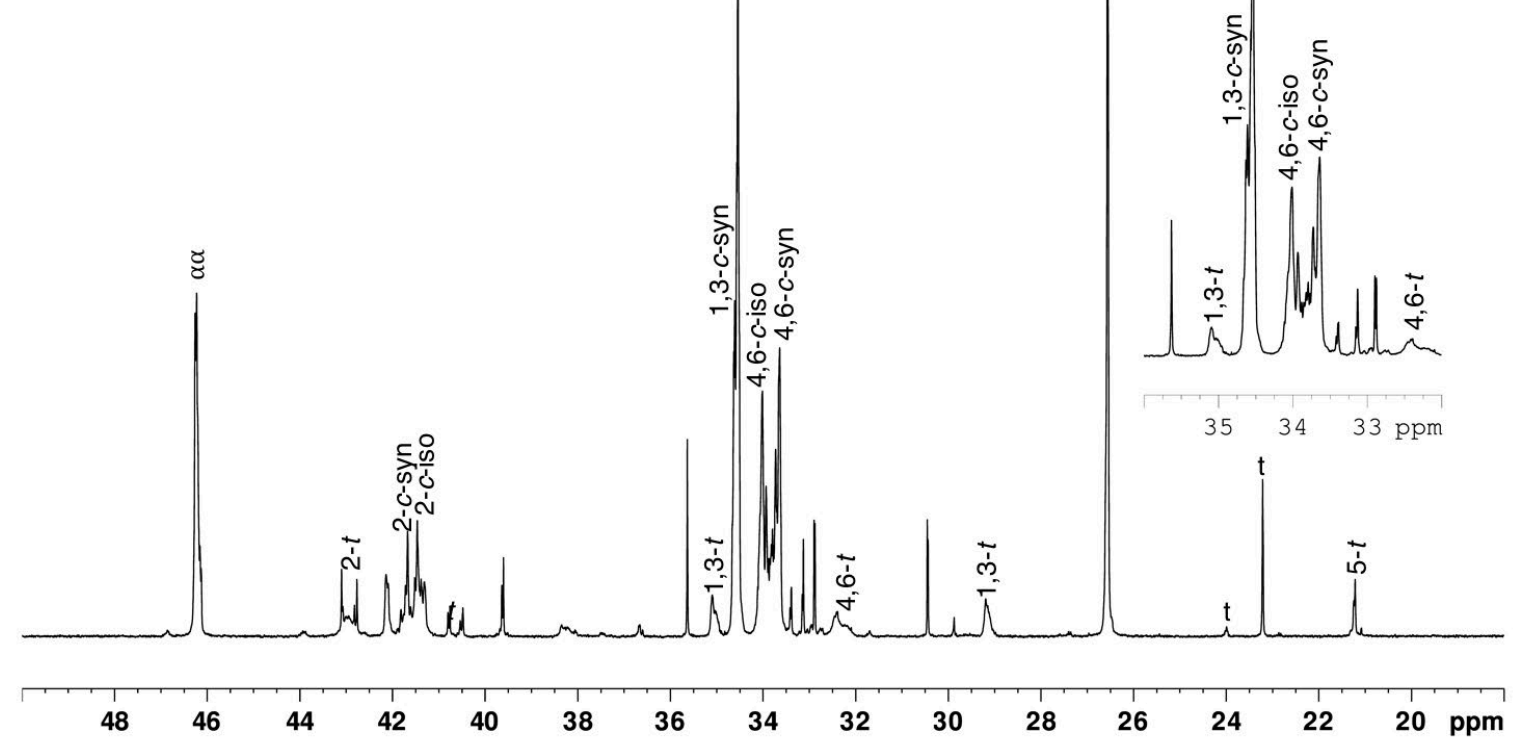

Figure S11. A) ${ }^{1} \mathrm{H}$ NMR $\left(800 \mathrm{MHz}, \mathrm{CDCl}_{3}, 25^{\circ} \mathrm{C}\right)$ of $\mathrm{PMCH}$ from run 9. B) Partial ${ }^{13} \mathrm{C}\left\{{ }^{1} \mathrm{H}\right\}$ NMR (200 $\mathrm{MHz}, \mathrm{CDCl}_{3}, 25^{\circ} \mathrm{C}$ ) of $\mathrm{PMCH}$ from run 9; $\mathrm{X}$ denotes solvent peak. 
A

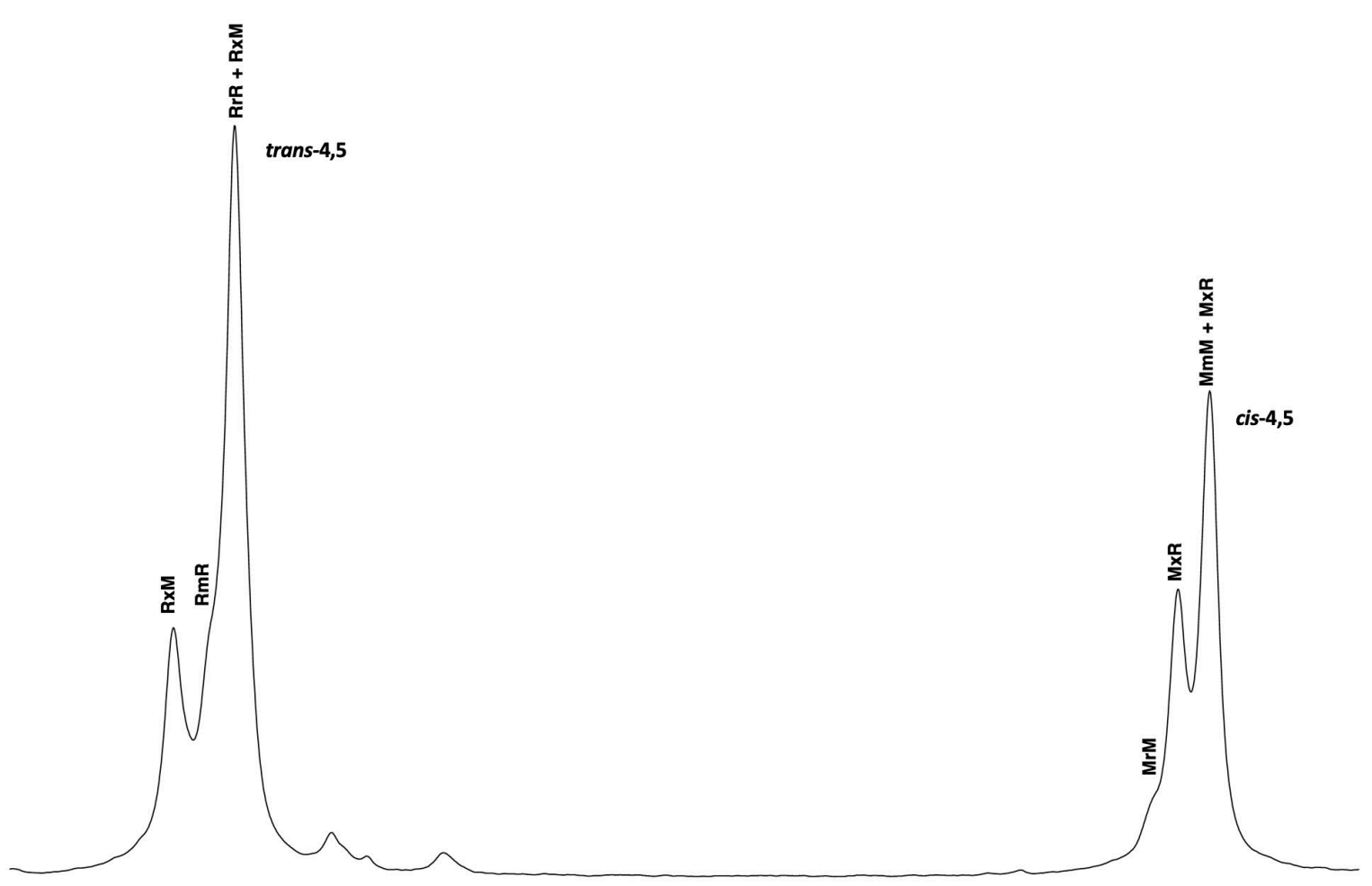

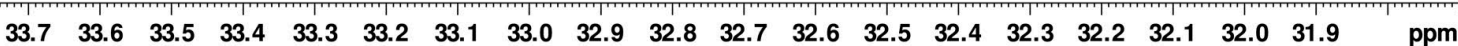

B

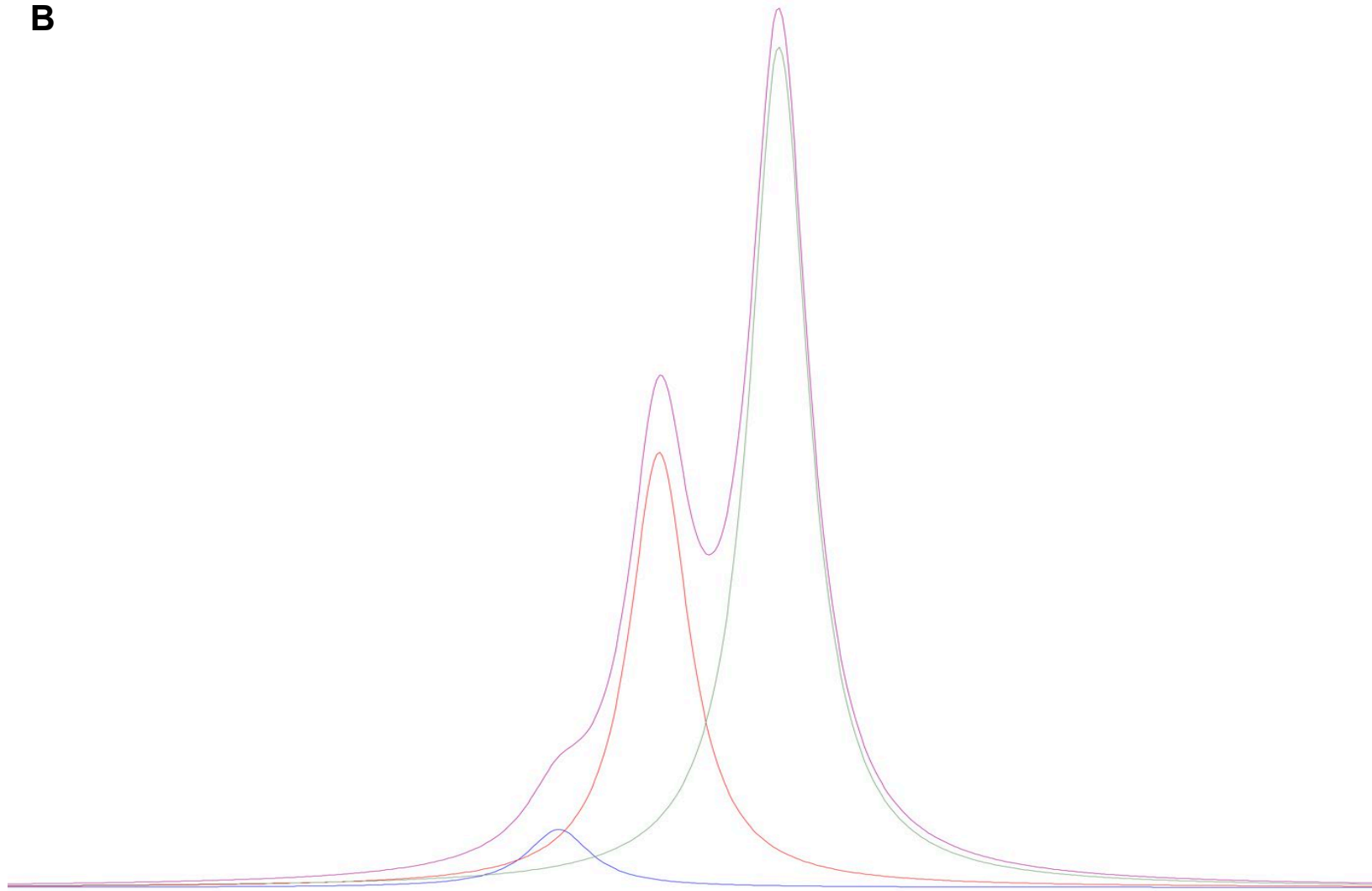

Figure S12. A) Partial ${ }^{13} \mathrm{C}\left\{{ }^{1} \mathrm{H}\right\} \mathrm{NMR}\left(100 \mathrm{MHz}, \mathrm{CDCl} l_{3}, 25^{\circ} \mathrm{C}\right)$ of the trans $-4,5$ and cis $-4,5$ resonances of PMCP from run 1 with stereochemical assignments. B) Deconvoluted cis-4,5 resonances of PMCP from run 1. 
A

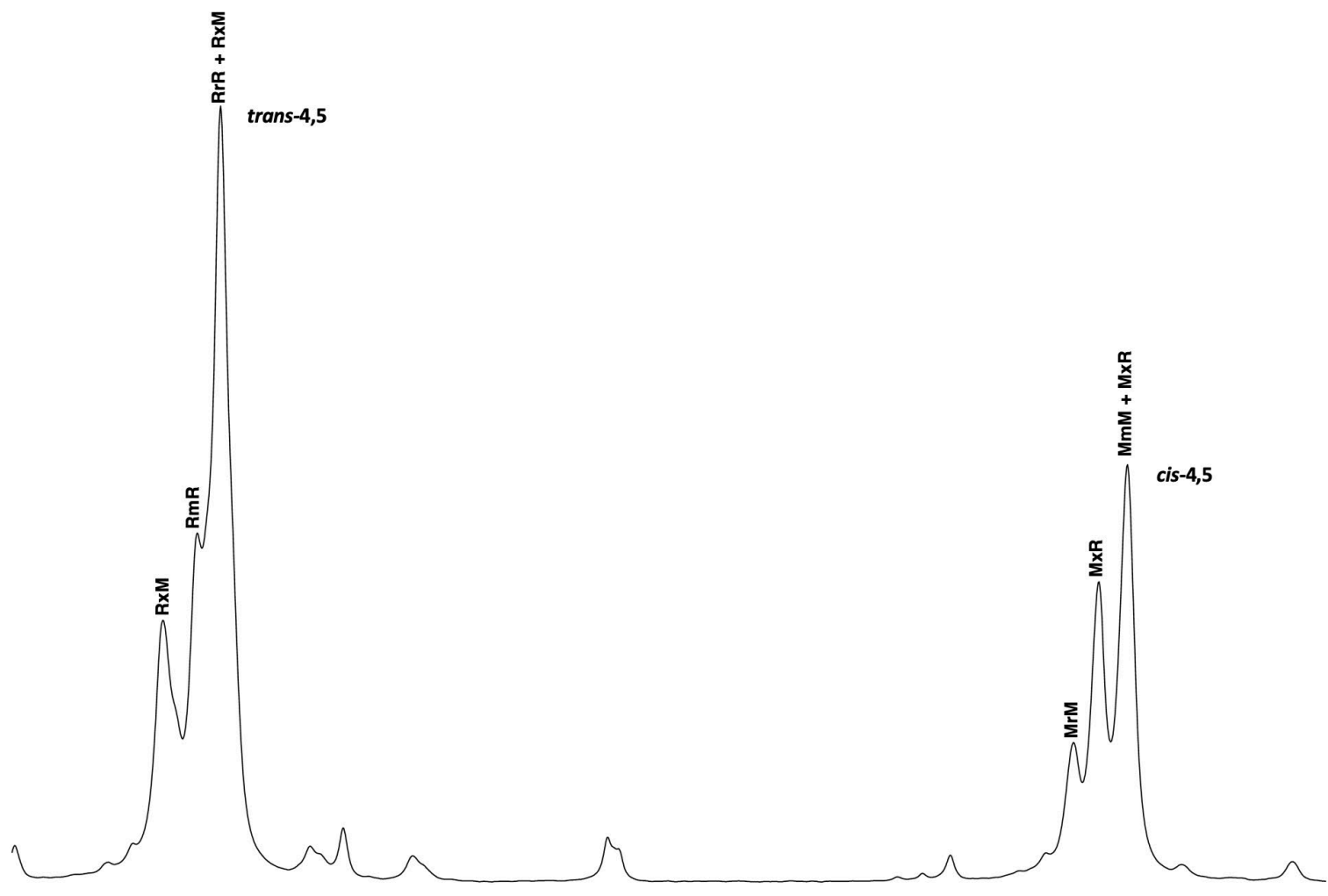

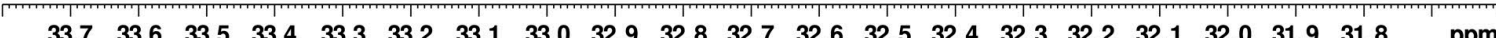

B

$\begin{array}{lllllllllllllllllllll}33.7 & 33.6 & 33.5 & 33.4 & 33.3 & 33.2 & 33.1 & 33.0 & 32.9 & 32.8 & 32.7 & 32.6 & 32.5 & 32.4 & 32.3 & 32.2 & 32.1 & 32.0 & 31.9 & 31.8 & \mathrm{ppm}\end{array}$

Figure S13. A) Partial ${ }^{13} \mathrm{C}\left\{{ }^{1} \mathrm{H}\right\}$ NMR $\left(100 \mathrm{MHz}, \mathrm{CDCl}_{3}, 25^{\circ} \mathrm{C}\right)$ of the trans $-4,5$ and cis $-4,5$ resonances of PMCP from run 2 with stereochemical assignments. B) Deconvoluted cis- 4,5 resonances of PMCP from run 2. 


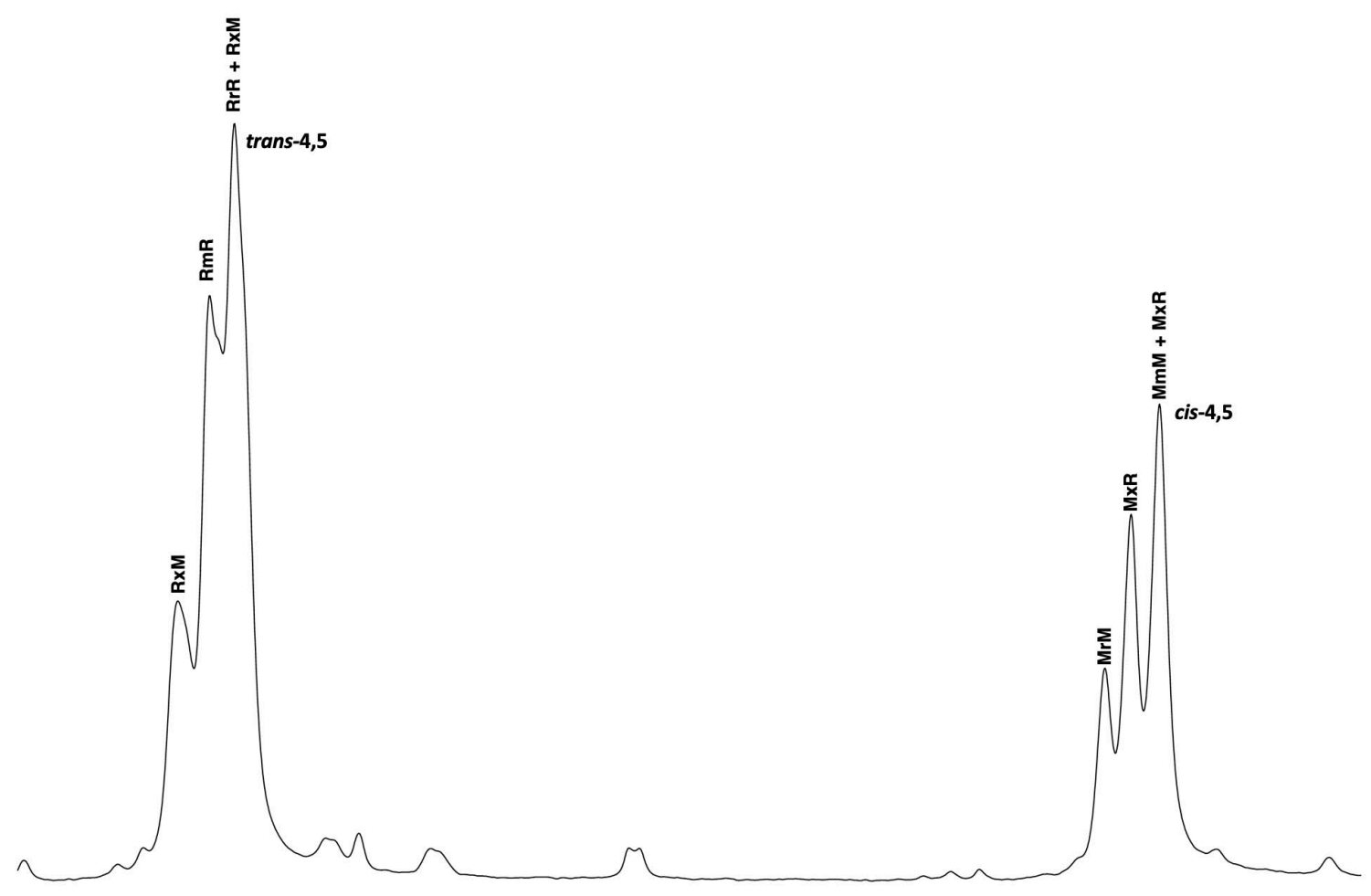

$\begin{array}{llllllllllllllllllllllllllll}33.7 & 33.6 & 33.5 & 33.4 & 33.3 & 33.2 & 33.1 & 33.0 & 32.9 & 32.8 & 32.7 & 32.6 & 32.5 & 32.4 & 32.3 & 32.2 & 32.1 & 32.0 & 31.9 & 31.8 & \mathrm{ppm}\end{array}$

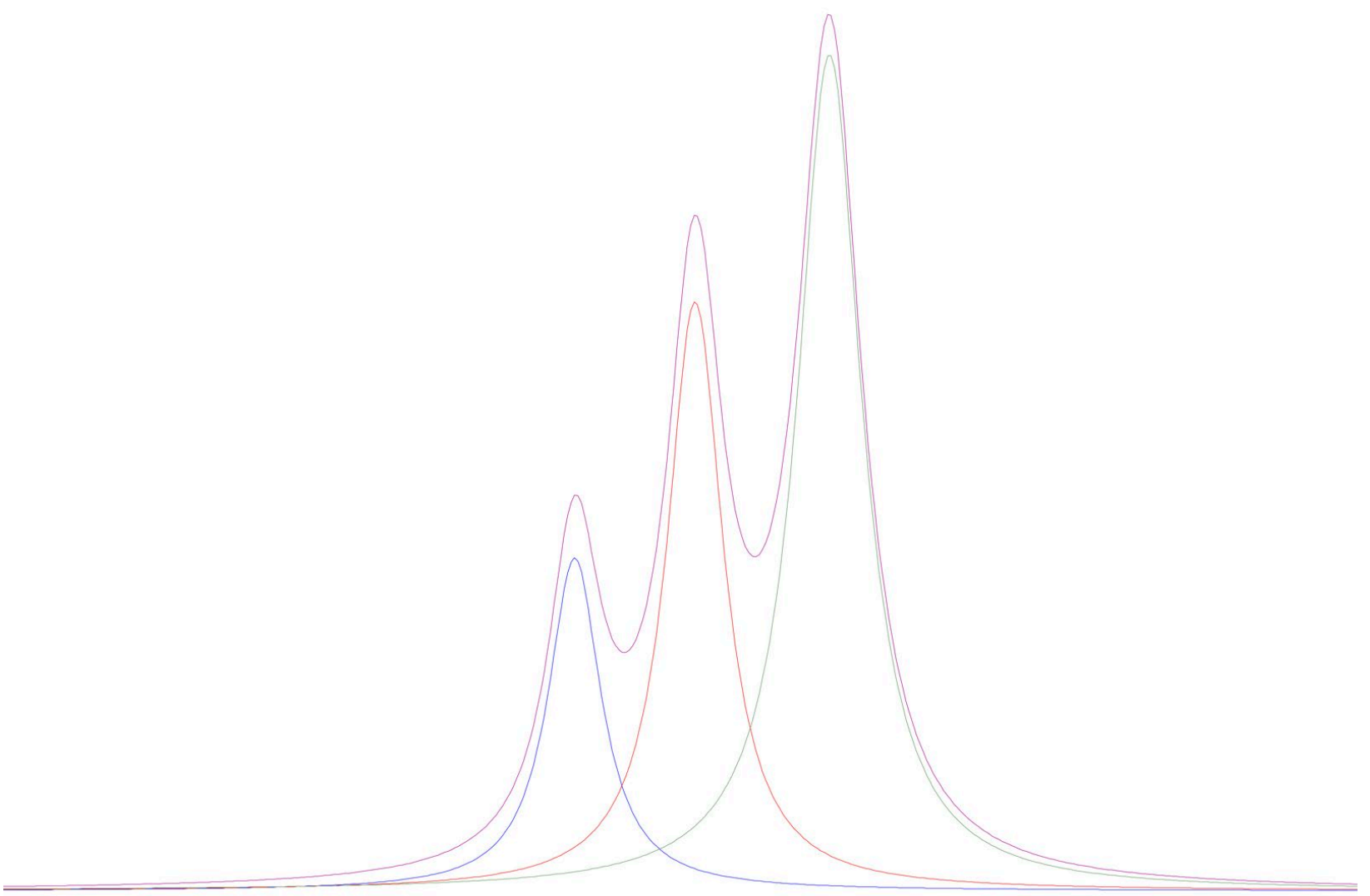

Figure S14. A) Partial ${ }^{13} \mathrm{C}\left\{{ }^{1} \mathrm{H}\right\} \mathrm{NMR}\left(100 \mathrm{MHz}, \mathrm{CDCl}_{3}, 25^{\circ} \mathrm{C}\right)$ of the trans $-4,5$ and cis- 4,5 resonances of PMCP from run 3 with stereochemical assignments. B) Deconvoluted cis- 4,5 resonances of PMCP from run 3. 


\begin{tabular}{|c|c|c|c|c|c|c|c|}
\hline \multirow[t]{2}{*}{ Fit } & \multicolumn{2}{|c|}{ Frequency } & \multicolumn{2}{|c|}{ Width } & \multirow[t]{2}{*}{ Intensity } & \multirow[t]{2}{*}{ Area } & \multirow{2}{*}{$\begin{array}{l}\text { \%Lor. } \\
\text { chisq }\end{array}$} \\
\hline & ppm & $\mathrm{Hz}$ & ppm & $\mathrm{Hz}$ & & & \\
\hline \multirow[t]{2}{*}{1} & & & & & & & $8.8 e+14$ \\
\hline & 31.893 & 3208.83 & 0.03254 & 3.274 & 0.651 & 27.248 & 100.00 \\
\hline \multirow[t]{2}{*}{ STD : } & 0.001 & 0.09 & 0.00310 & 0.312 & 0.032 & & \\
\hline & 31.851 & 3204.61 & 0.03063 & 3.082 & 4.896 & 192.901 & 100.00 \\
\hline \multirow[t]{2}{*}{ STD : } & 0.000 & 0.01 & 0.00047 & 0.048 & 0.033 & & \\
\hline & 31.801 & 3199.58 & 0.03006 & 3.024 & 9.460 & 365.772 & 100.00 \\
\hline STD : & 0.000 & 0.01 & 0.00018 & 0.018 & 0.032 & & \\
\hline \multirow[t]{2}{*}{ Fit } & \multicolumn{2}{|c|}{ Frequency } & \multicolumn{2}{|c|}{ Width } & \multirow[t]{2}{*}{ Intensity } & \multirow[t]{2}{*}{ Area } & \multirow{2}{*}{$\begin{array}{l}\text { \%Lor. } \\
\text { chisq }\end{array}$} \\
\hline & ppm & $\mathrm{Hz}$ & ppm & $\mathrm{Hz}$ & & & \\
\hline \multirow[t]{2}{*}{1} & & & & & & \multicolumn{2}{|r|}{$1.5 e+15$} \\
\hline & 32.026 & 3222.18 & 0.02750 & 2.767 & 2.125 & 75.193 & 100.00 \\
\hline \multirow[t]{2}{*}{ STD : } & 0.000 & 0.04 & 0.00127 & 0.128 & 0.057 & & \\
\hline & 31.982 & 3217.84 & 0.02477 & 2.492 & 5.167 & 164.617 & 100.00 \\
\hline \multirow[t]{2}{*}{ STD : } & 0.000 & 0.02 & 0.00057 & 0.057 & 0.060 & & \\
\hline & 31.934 & 3212.96 & 0.02570 & 2.586 & 7.945 & 262.667 & 100.00 \\
\hline STD : & 0.000 & 0.01 & 0.00031 & 0.031 & 0.058 & & \\
\hline
\end{tabular}

\begin{tabular}{|c|c|c|c|c|c|c|c|}
\hline \multirow[t]{2}{*}{ Fit } & \multicolumn{2}{|c|}{ Frequency } & \multicolumn{2}{|c|}{ Width } & \multirow[t]{2}{*}{ Intensity } & \multirow[t]{2}{*}{ Area } & \multirow{2}{*}{$\begin{array}{l}\text { \%Lor. } \\
\text { chisq }\end{array}$} \\
\hline & ppm & $\mathrm{Hz}$ & ppm & $\mathrm{Hz}$ & & & \\
\hline 1 & & & & & & & $1.4 \mathrm{e}+15$ \\
\hline & 32.021 & 3221.72 & 0.02296 & 2.310 & 2.683 & 79.241 & 100.00 \\
\hline STD : & 0.000 & 0.02 & 0.00055 & 0.055 & 0.038 & & \\
\hline & 31.978 & 3217.41 & 0.02432 & 2.447 & 4.752 & 148.669 & 100.00 \\
\hline STD : & 0.000 & 0.01 & 0.00037 & 0.037 & 0.037 & & \\
\hline & 31.930 & 3212.59 & 0.02783 & 2.800 & 6.744 & 241.425 & 100.00 \\
\hline STD : & 0.000 & 0.01 & 0.00024 & 0.024 & 0.035 & & \\
\hline
\end{tabular}

Figure S15. Data produced from the deconvolution the cis-4,5 resonances of PMCP from run 1 (top), run 2 (middle), and run 3 (bottom) using a Lorentzian fit. 


\section{Simulation of ${ }^{13} \mathrm{C}$ NMR for PMCP}

Tetrad intensities were calculated for the two parameters, $\alpha$ and $\sigma$, through the following relationships. ${ }^{4}{ }^{13} \mathrm{C}$ NMR spectra for Figure 1 were then simulated using gNMR Version

4.1

$\begin{array}{ll}\text { TETRAD } & \text { PROBABILTY } \\ \operatorname{RrR} & (1-\sigma)^{2}(\alpha)^{2}+(1-\sigma)^{2}(1-\alpha)^{2} \\ \operatorname{RmR} & 2(1-\sigma)^{2}(1-\alpha)^{2} \alpha \\ \mathrm{MrM} & 2 \sigma^{2}(1-\alpha) \alpha \\ \mathrm{MmM} & \sigma^{2} \alpha^{2}+\sigma^{2}(1-\alpha)^{2} \\ \operatorname{RrM} & \sigma(1-\sigma) / 2( \\ \mathrm{RmM} & \sigma(1-\sigma) / 2 \\ \mathrm{MrR} & \sigma(1-\sigma) / 2 \\ \mathrm{MmR} & \sigma(1-\sigma) / 2\end{array}$

For 13C NMR analysis of PMCP (run 1, Table 1): $\alpha=0.93 ; \sigma=0.35$

CHEMICAL SHIFT (ppm)

31.94

31.98

32.01

33.35

33.38

33.43
TETRAD

$M m M+M x R$

$M \times R$

MrM

RrR+RxM

$\mathrm{RmR}$

$\mathrm{RxM}$
CALCD

22.03

11.38

1.59

48.12

5.50

11.38

For 13C NMR analysis of PMCP (run 2, Table 1): $\alpha=0.69 ; \sigma=0.35$

\section{CHEMICAL SHIFT TETRAD CALCD \\ (ppm)}

$\begin{array}{llr}31.94 & \text { MmM+MxR } & 18.38 \\ 31.98 & \text { MxR } & 11.38 \\ 32.01 & \text { MrM } & 5.24 \\ 33.35 & \text { RrR+RxM } & 35.55 \\ 33.38 & \text { RmR } & 18.07 \\ 33.43 & \text { RxM } & 11.38\end{array}$

For 13C NMR analysis of PMCP (run 1, Table 1): $\alpha=0.93 ; \sigma=0.35$

\section{CHEMICAL SHIFT TETRAD CALCD \\ (ppm)}

$\begin{array}{llr}31.94 & \text { MmM+MxR } & 22.03 \\ 31.98 & \text { MxR } & 11.38 \\ 32.01 & \text { MrM } & 1.59 \\ 33.35 & \text { RrR+RxM } & 48.12 \\ 33.38 & \text { RmR } & 5.50 \\ 33.43 & \text { RxM } & 11.38\end{array}$




\section{Supporting XRD Traces}

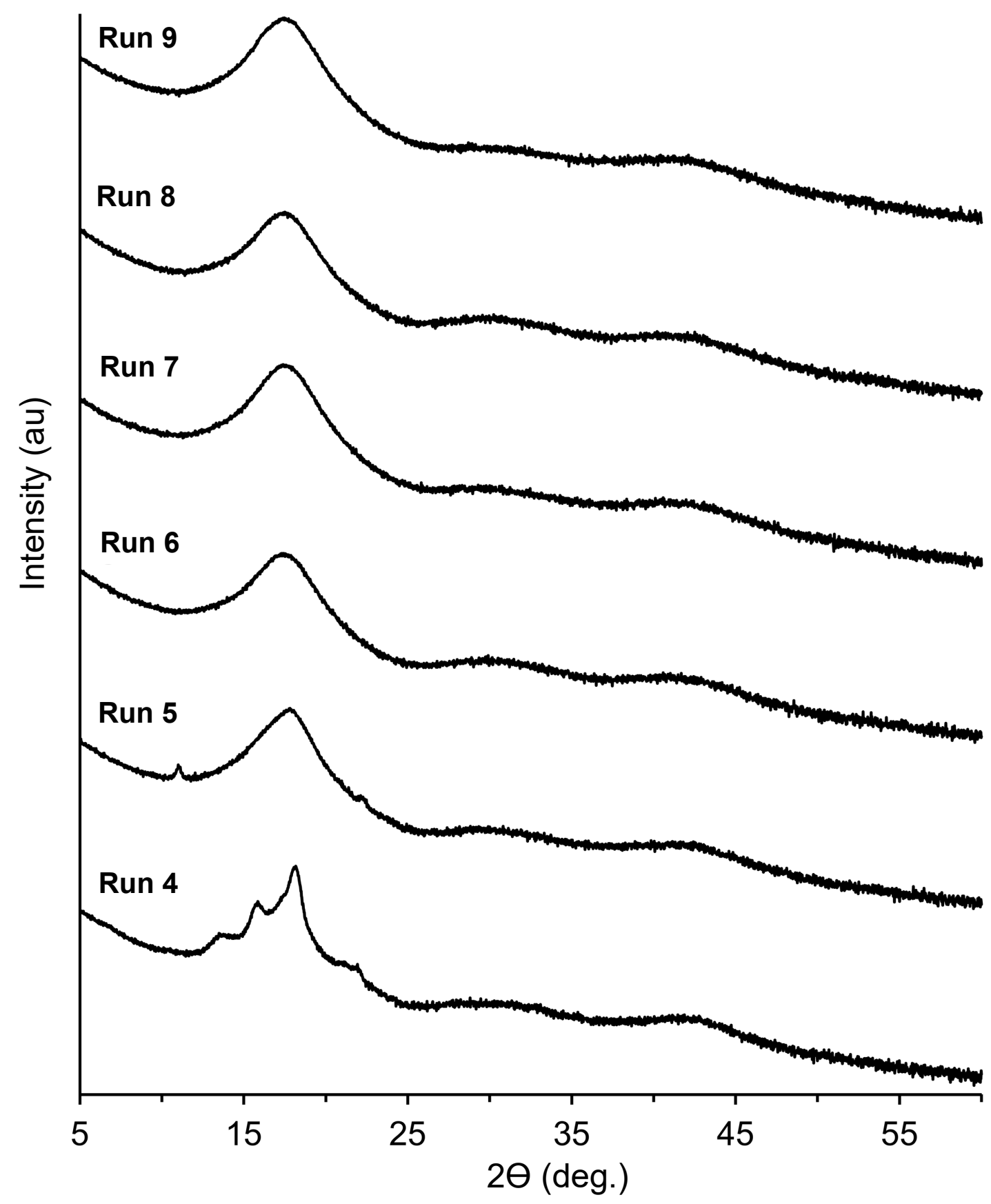

Figure S16. Stacked WAXD traces of the crystalline and amorphous phases of poly(1,3-methylenecyclohexane) from run 4 (bottom) to run 9 (top). 


\section{Supporting DSC Traces}

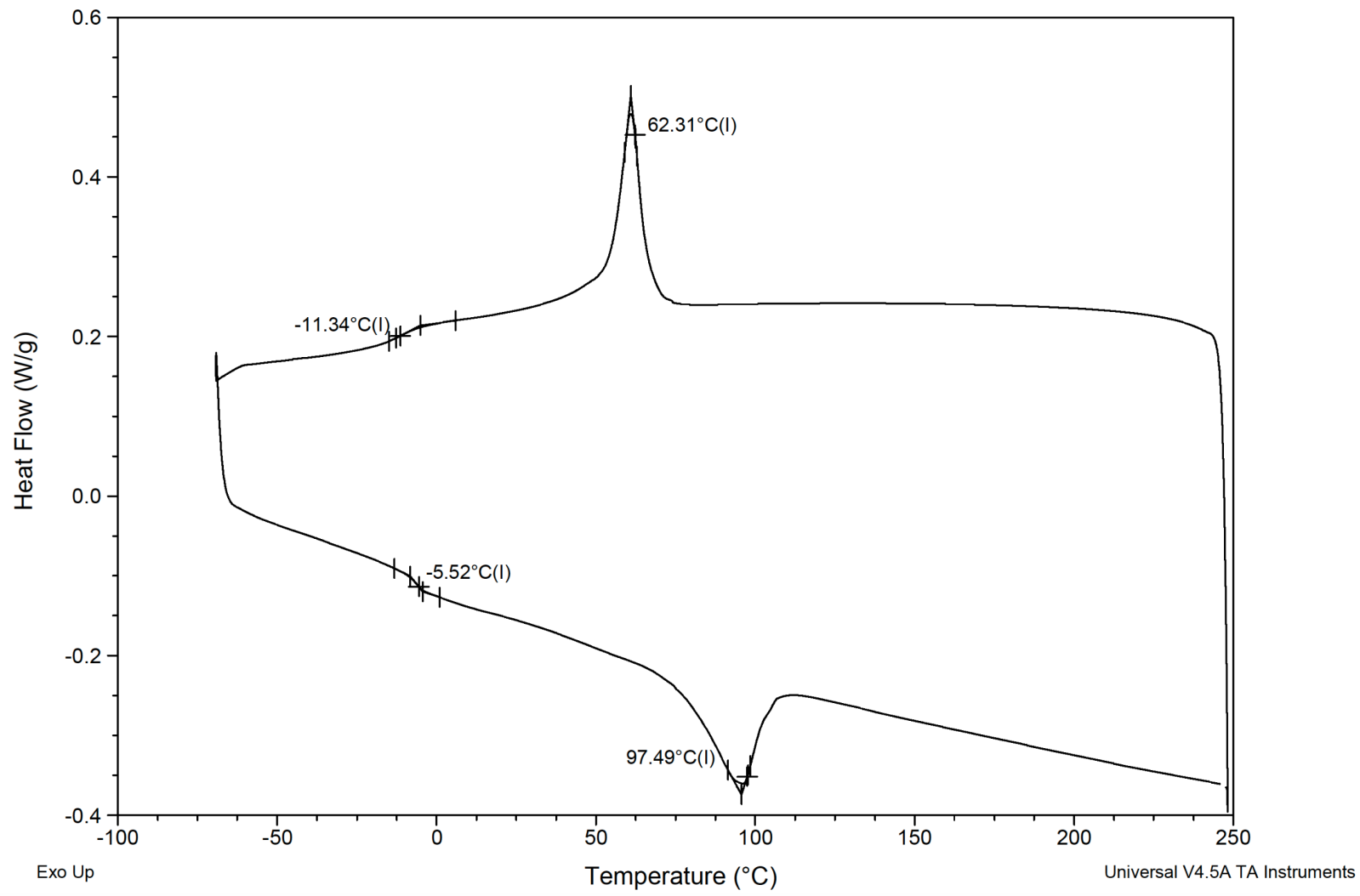

Figure S17. DSC trace of the second heating cycle and cooling cycle of PMCP from run 1. 


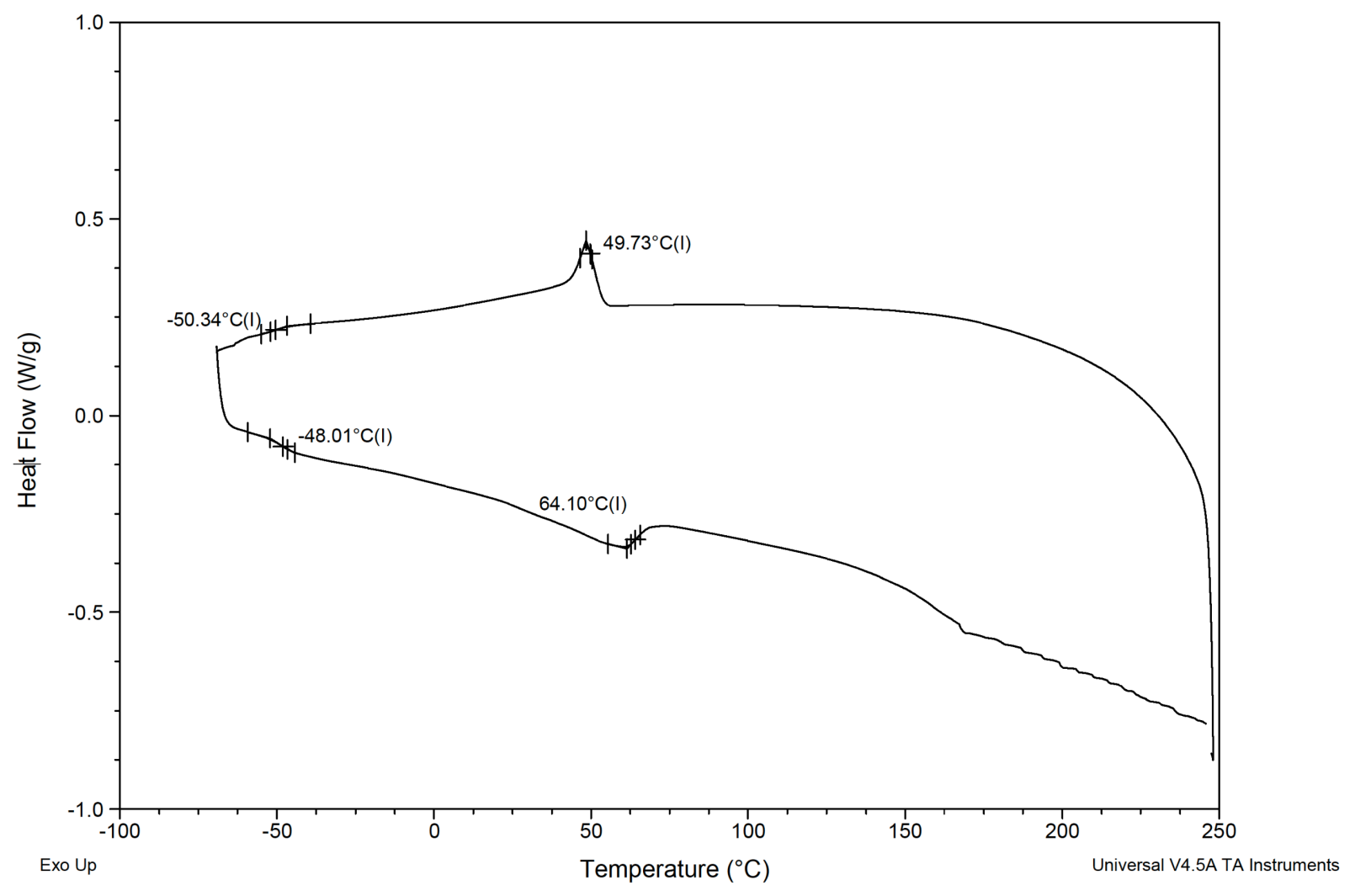

Figure S18. DSC trace of the second heating cycle and cooling cycle PMCP from run 2. 


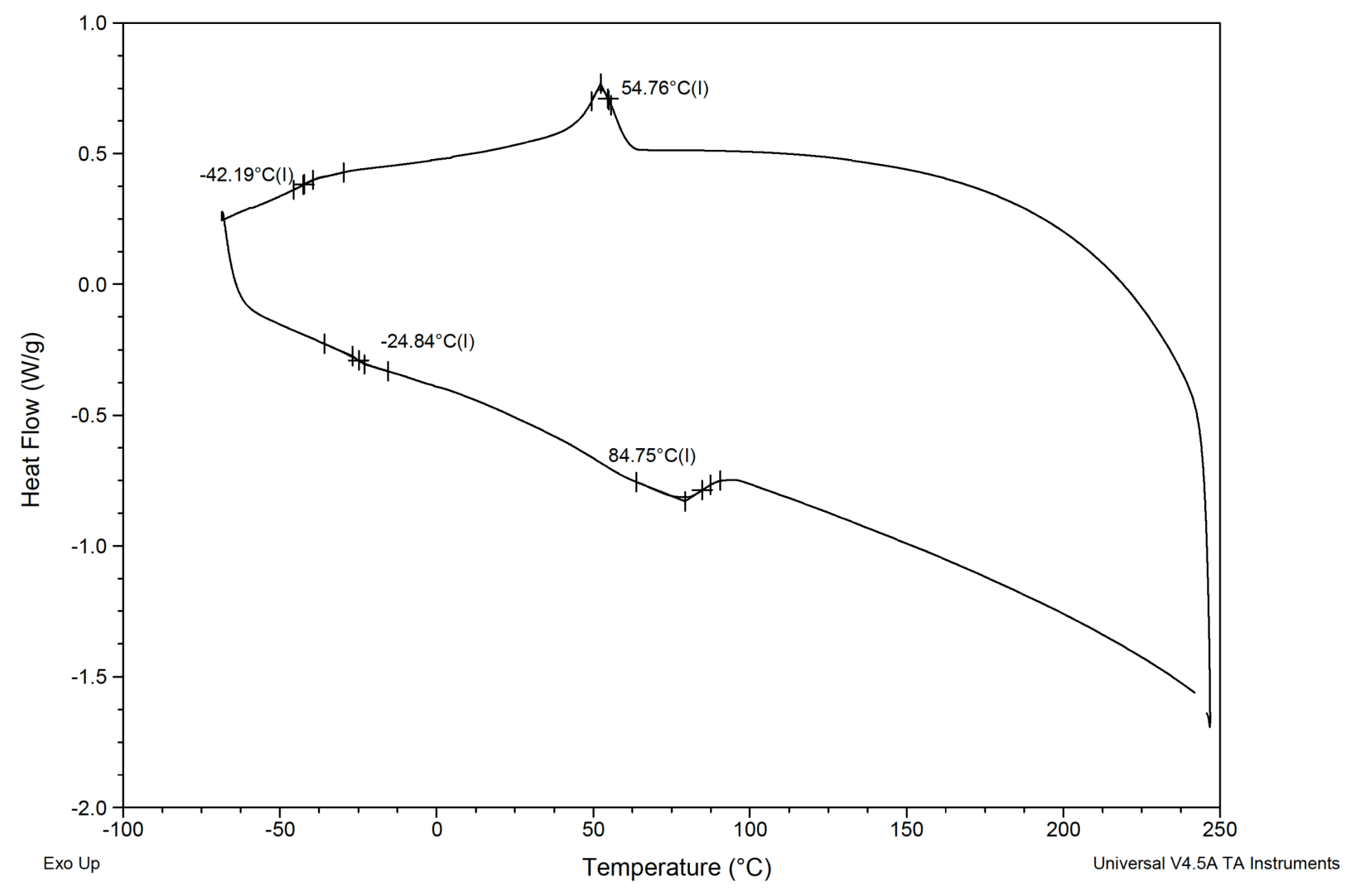

Figure S19. DSC trace of the second heating cycle and cooling cycle of PMCP from run 3. 


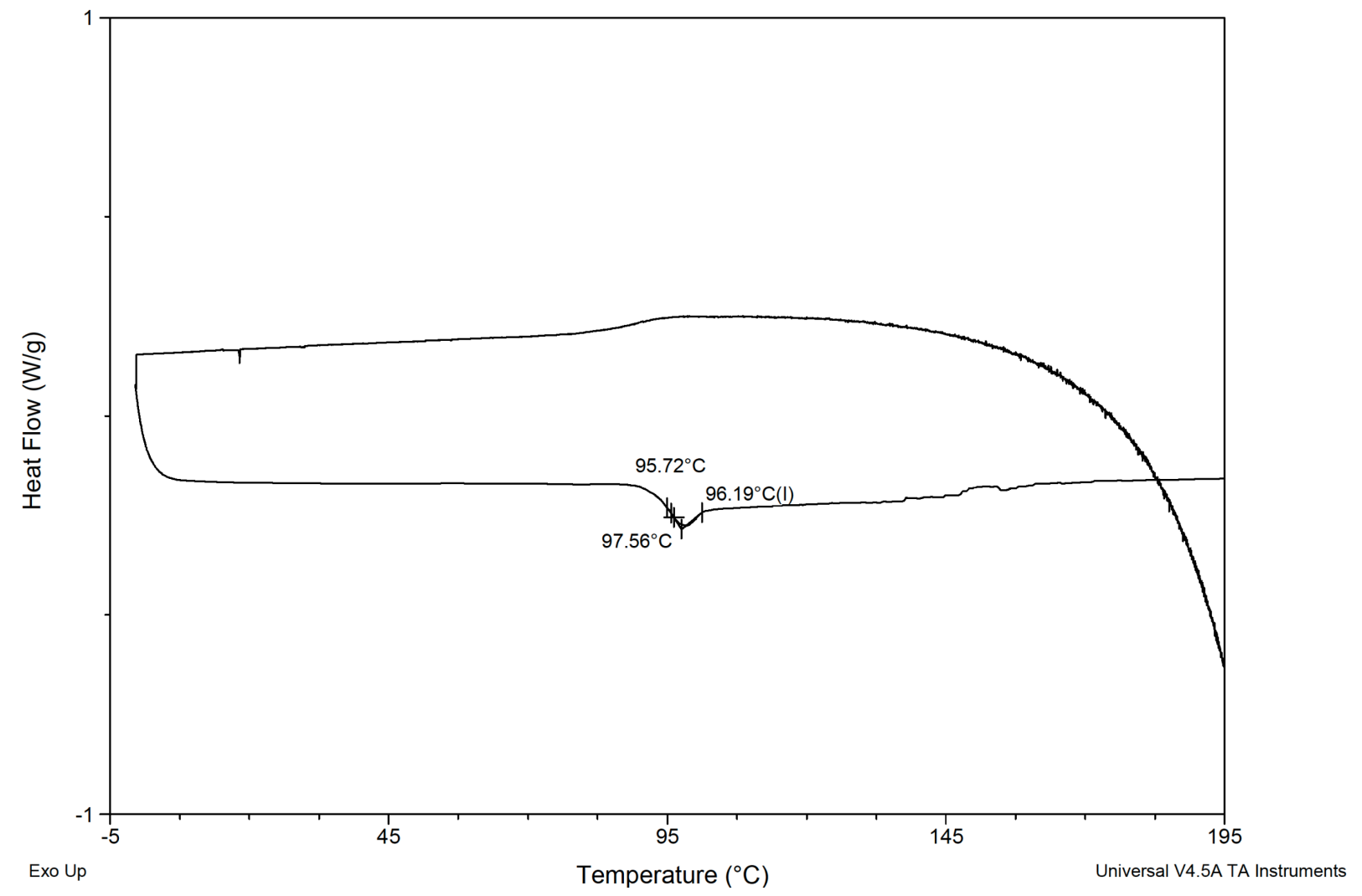

Figure S20. DSC trace of the second heating cycle and cooling cycle of $\mathrm{PMCH}$ from run 4. 


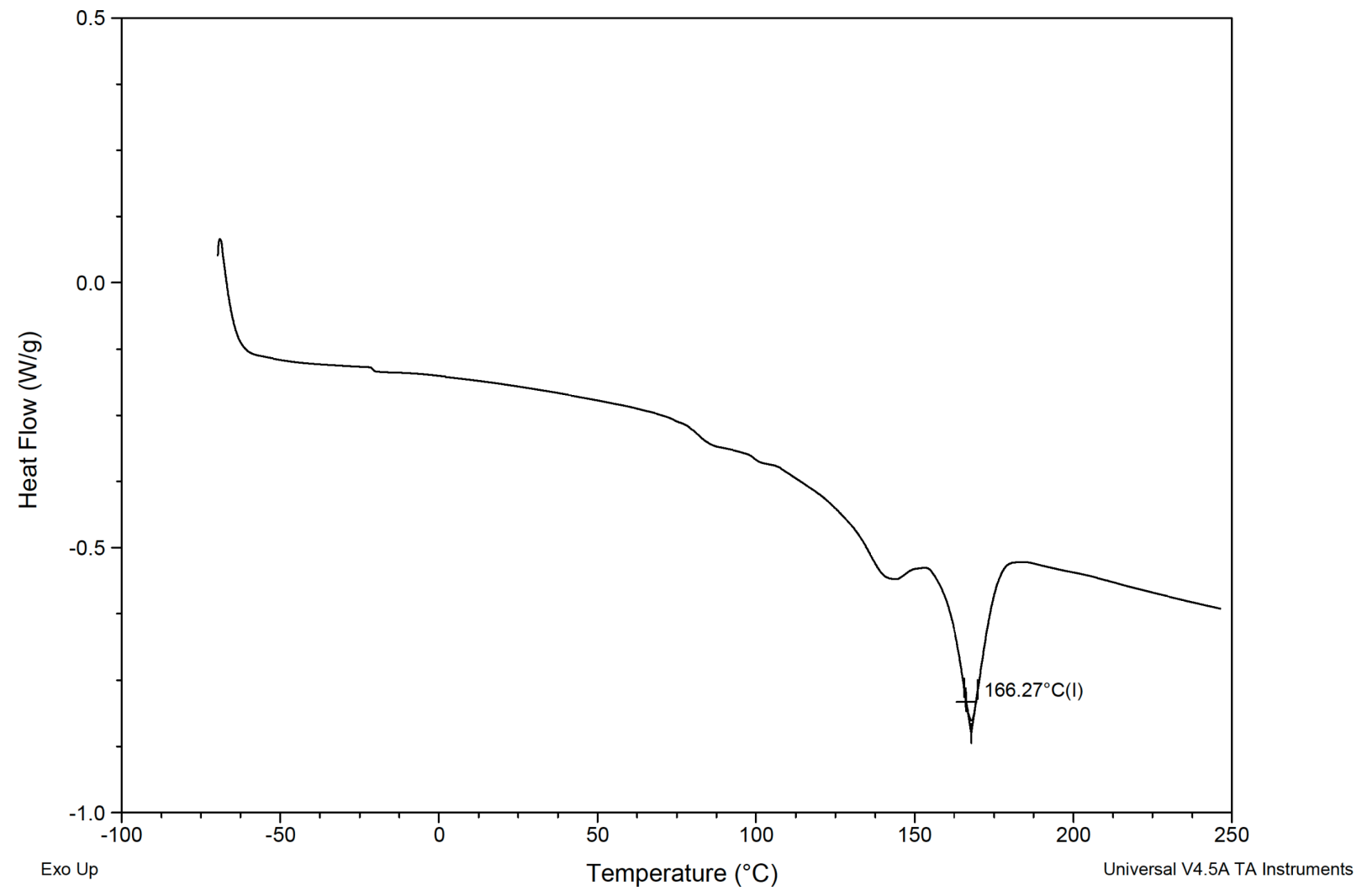

Figure S21. DSC trace of the first heating cycle of $\mathrm{PMCH}$ from run 4. 


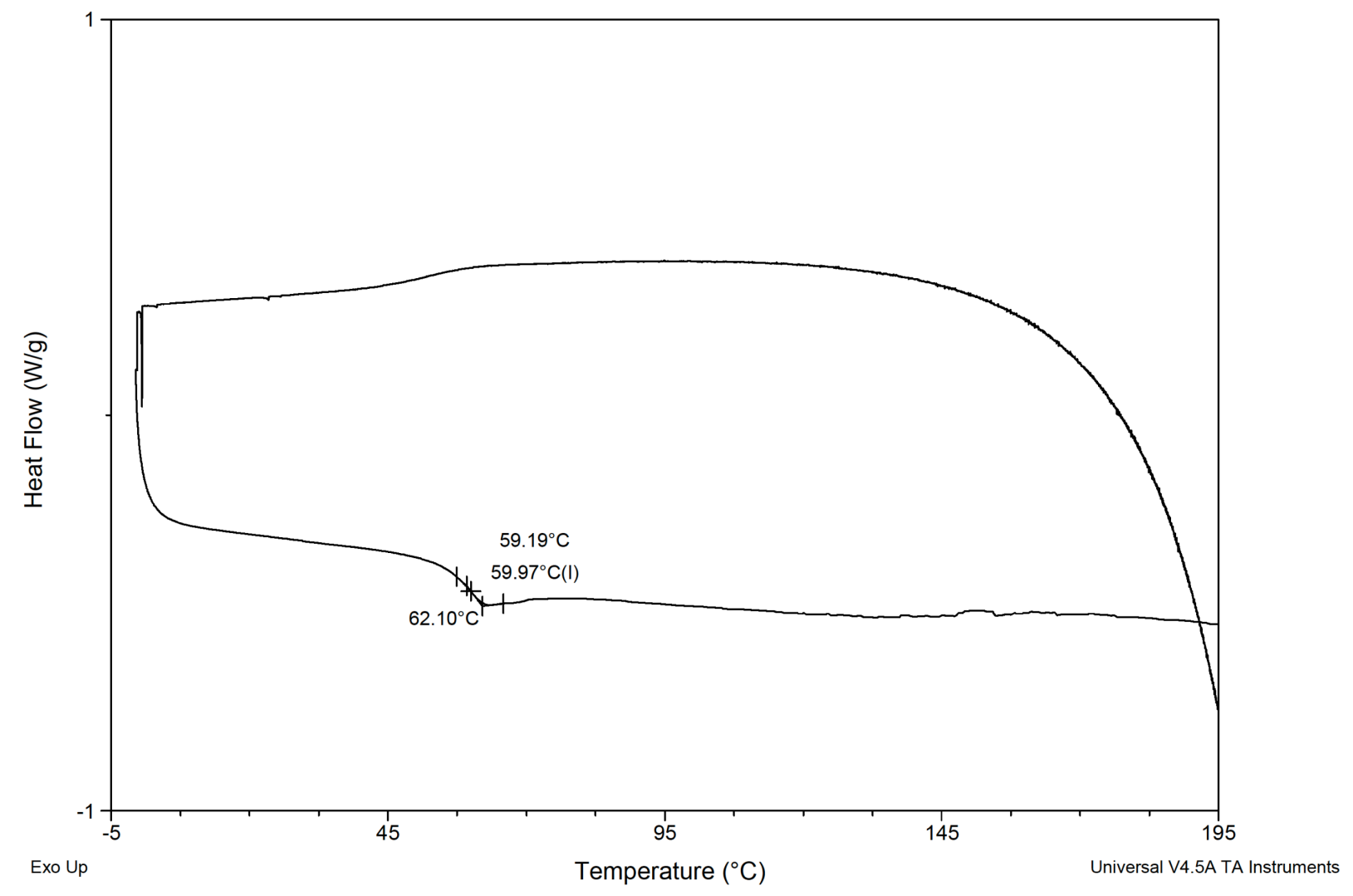

Figure S22. DSC trace of the second heating cycle and cooling cycle of PMCH from run 5. 


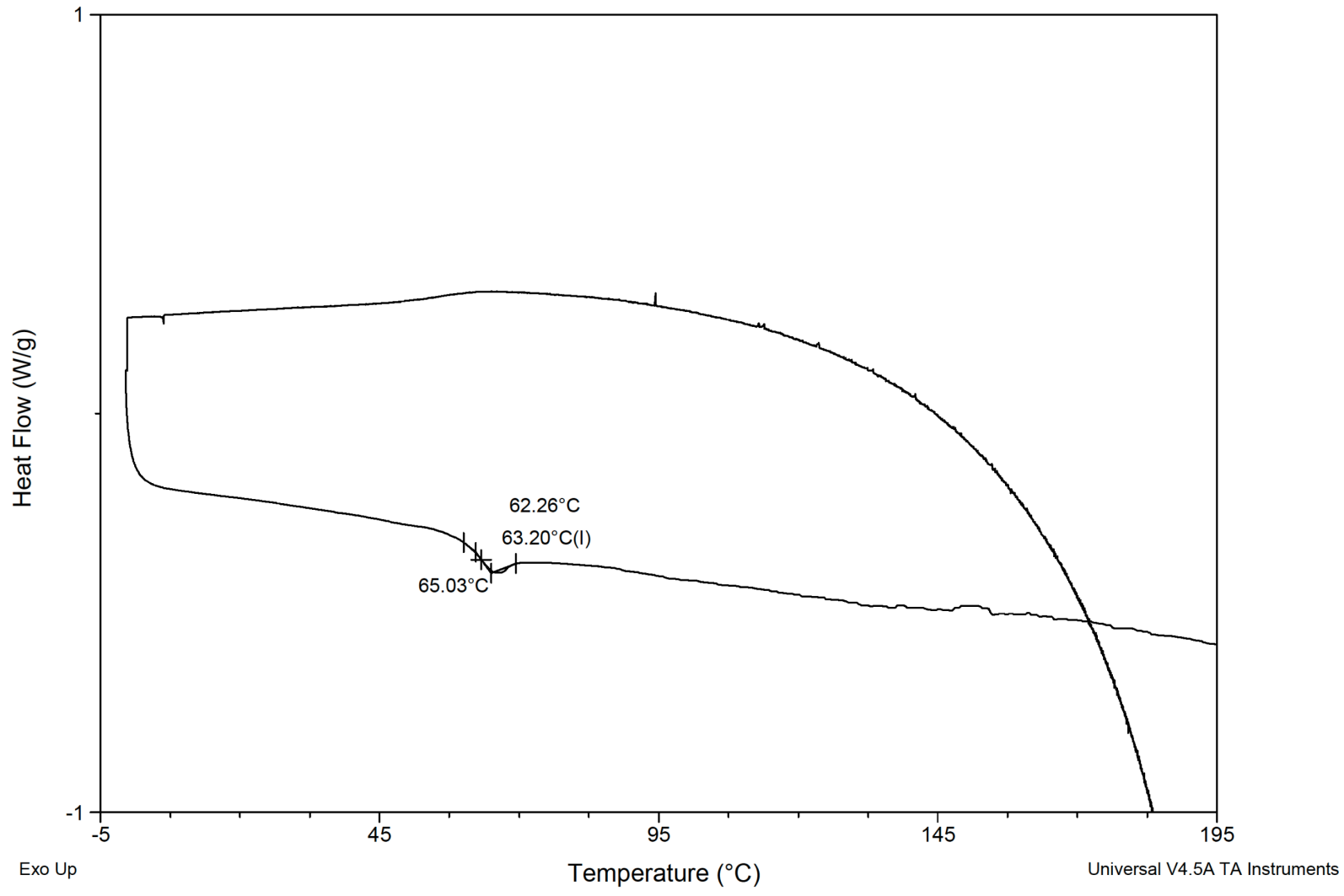

Figure S23. DSC trace of the second heating cycle and cooling cycle of $\mathrm{PMCH}$ from run 6. 


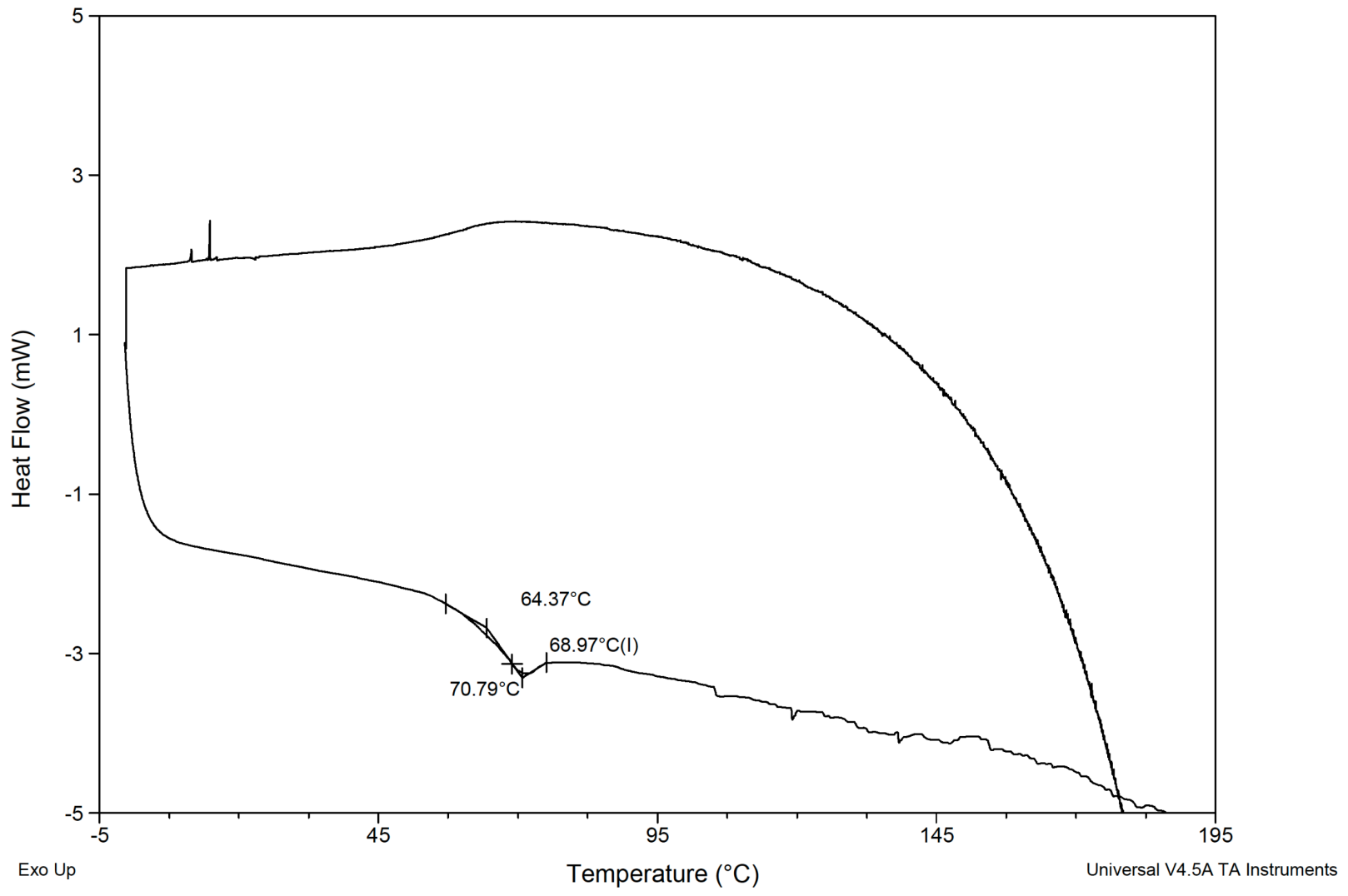

Figure S24. DSC trace of the second heating cycle and cooling cycle of PMCH from run 7. 


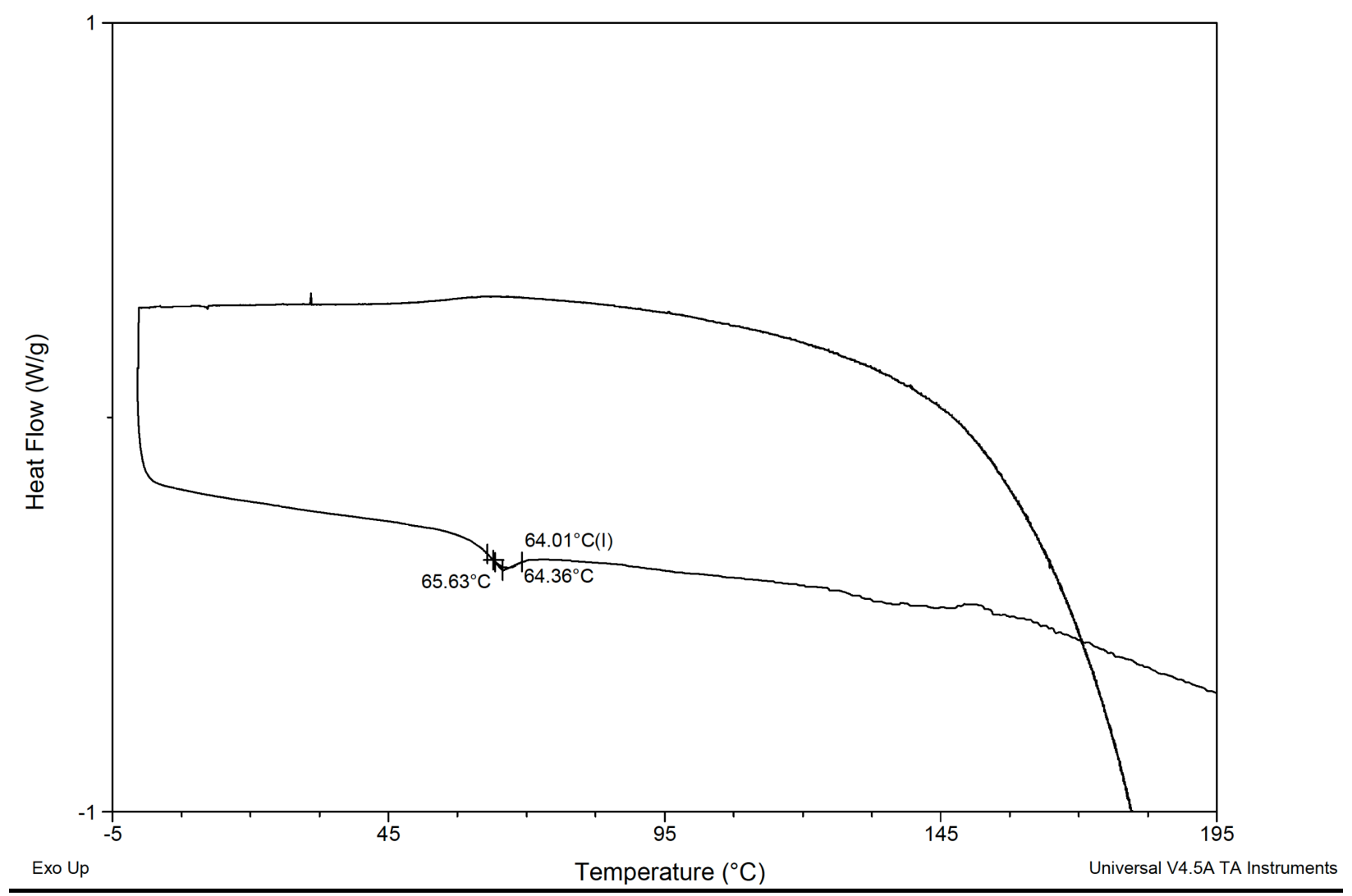

Figure S25. DSC trace of the second heating cycle and cooling cycle of $\mathrm{PMCH}$ from run 8. 


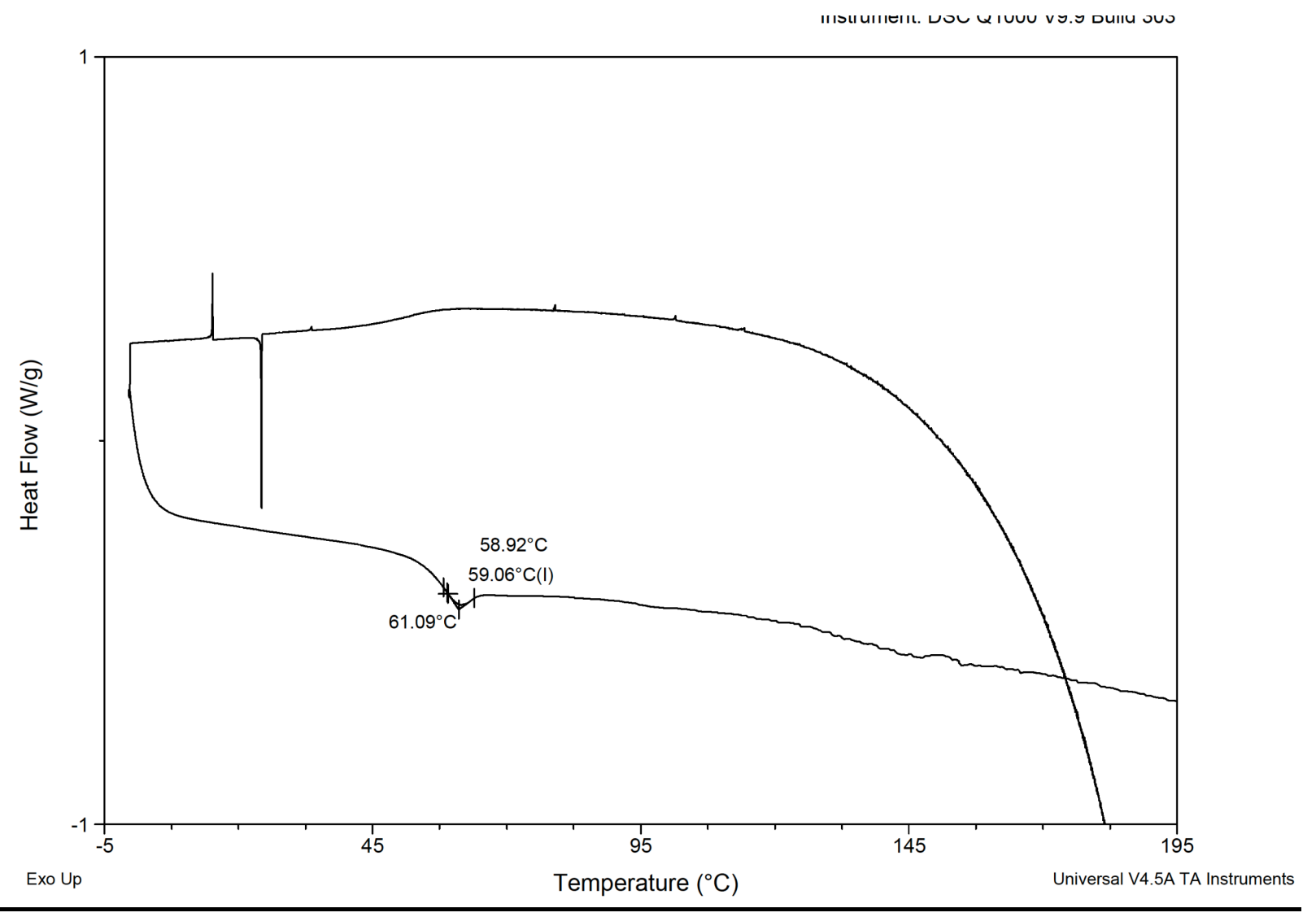

Figure S26. DSC trace of the second heating cycle and cooling cycle of PMCH from run 9. 
Quantitative ${ }^{13} \mathrm{C}$ NMR Analysis of $\mathrm{C}_{4,6}$-cis, iso to $\mathrm{C}_{4,6}$-cis, syn Ratio of PMCH from runs 6-9.

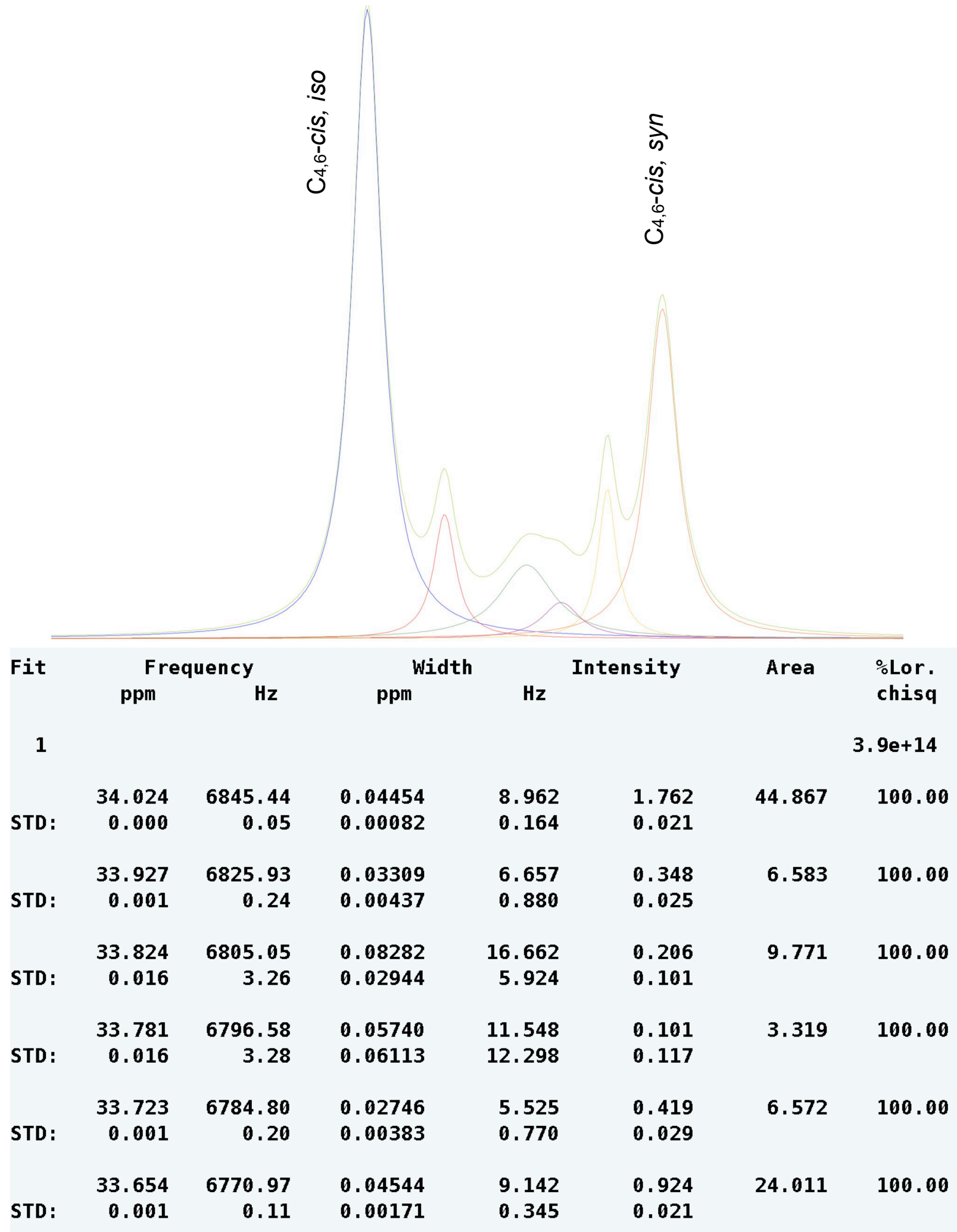

Figure S27. Data produced from the deconvolution the cis-4,6 resonances of $\mathrm{PMCH}$ from run 6 using a Lorentzian fit. 


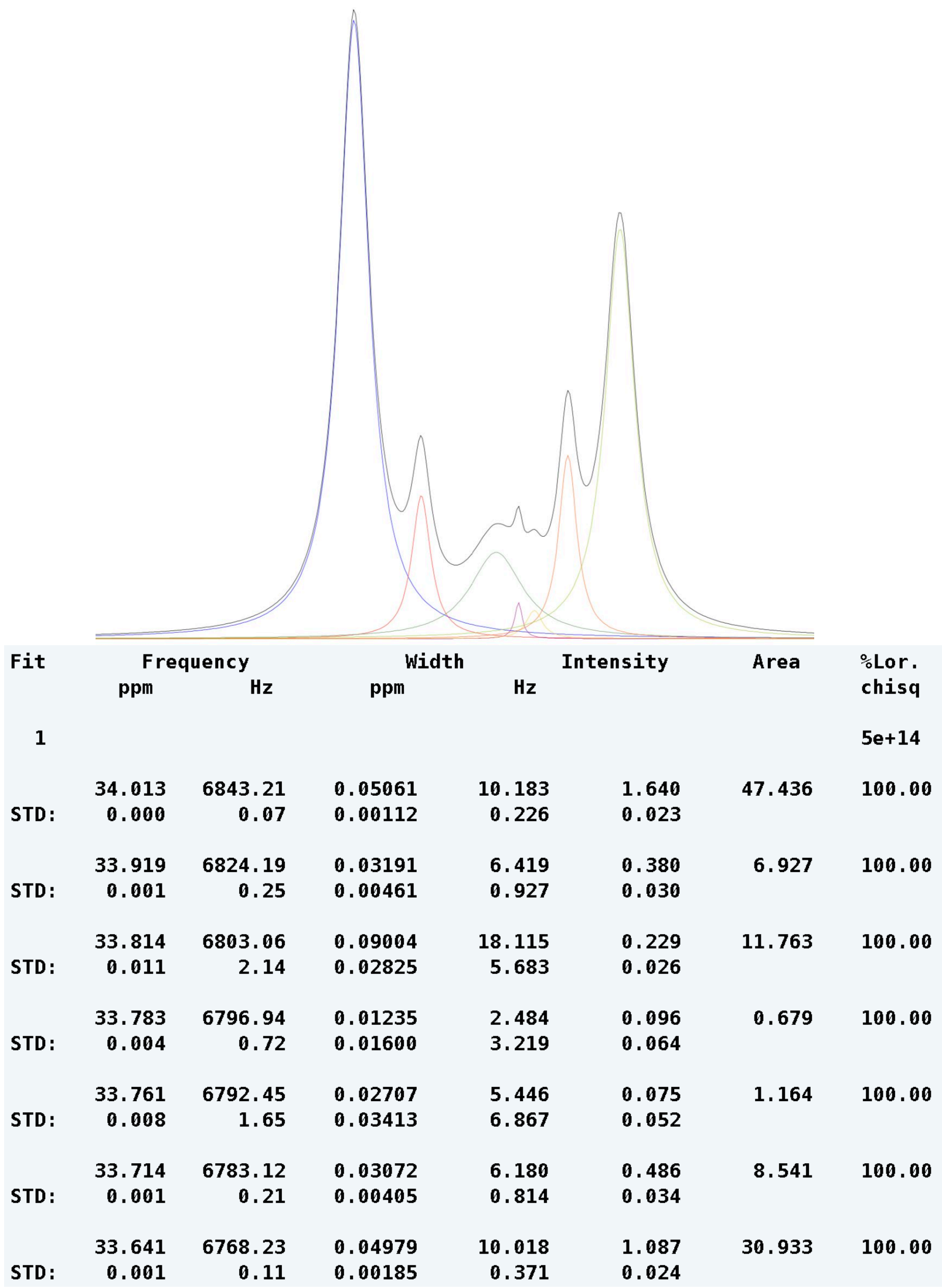

Figure S28. Data produced from the deconvolution the cis-4,6 resonances of $\mathrm{PMCH}$ from run 7 using a Lorentzian fit. 


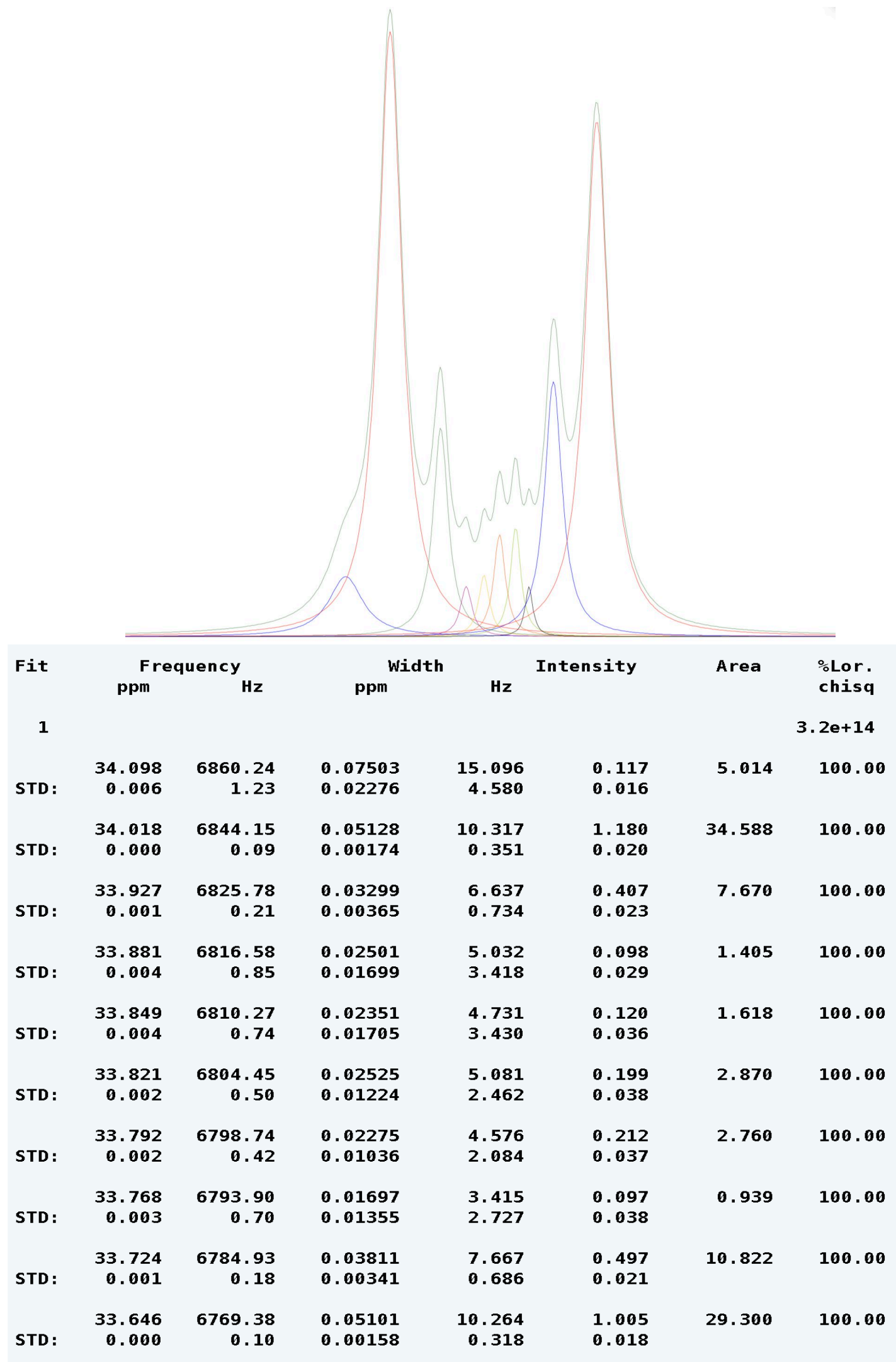

Figure S29. Data produced from the deconvolution the cis-4,6 resonances of $\mathrm{PMCH}$ from run 8 using a Lorentzian fit. 


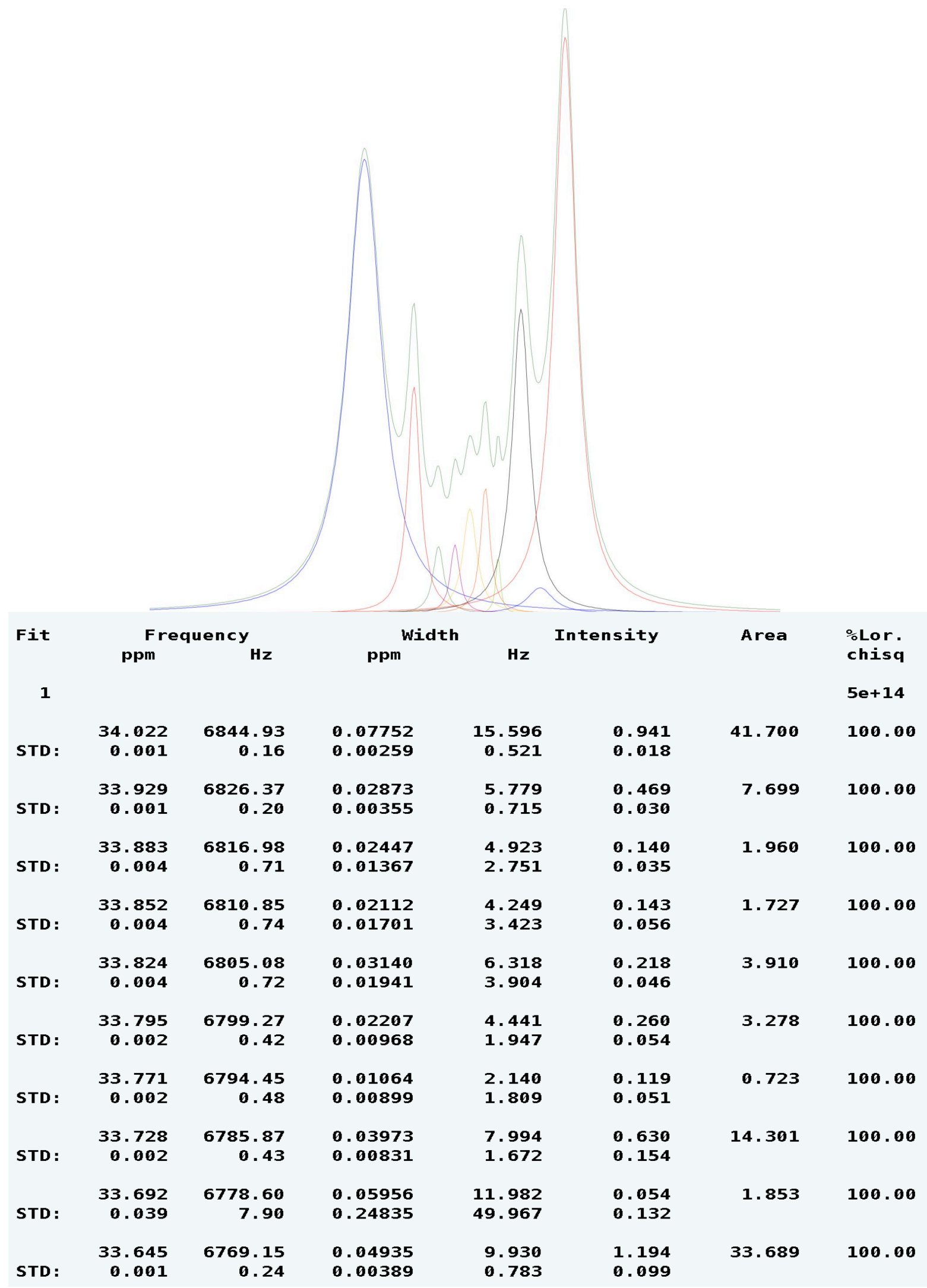

Figure S30. Data produced from the deconvolution the cis-4,6 resonances of $\mathrm{PMCH}$ from run 9 using a Lorentzian fit. 


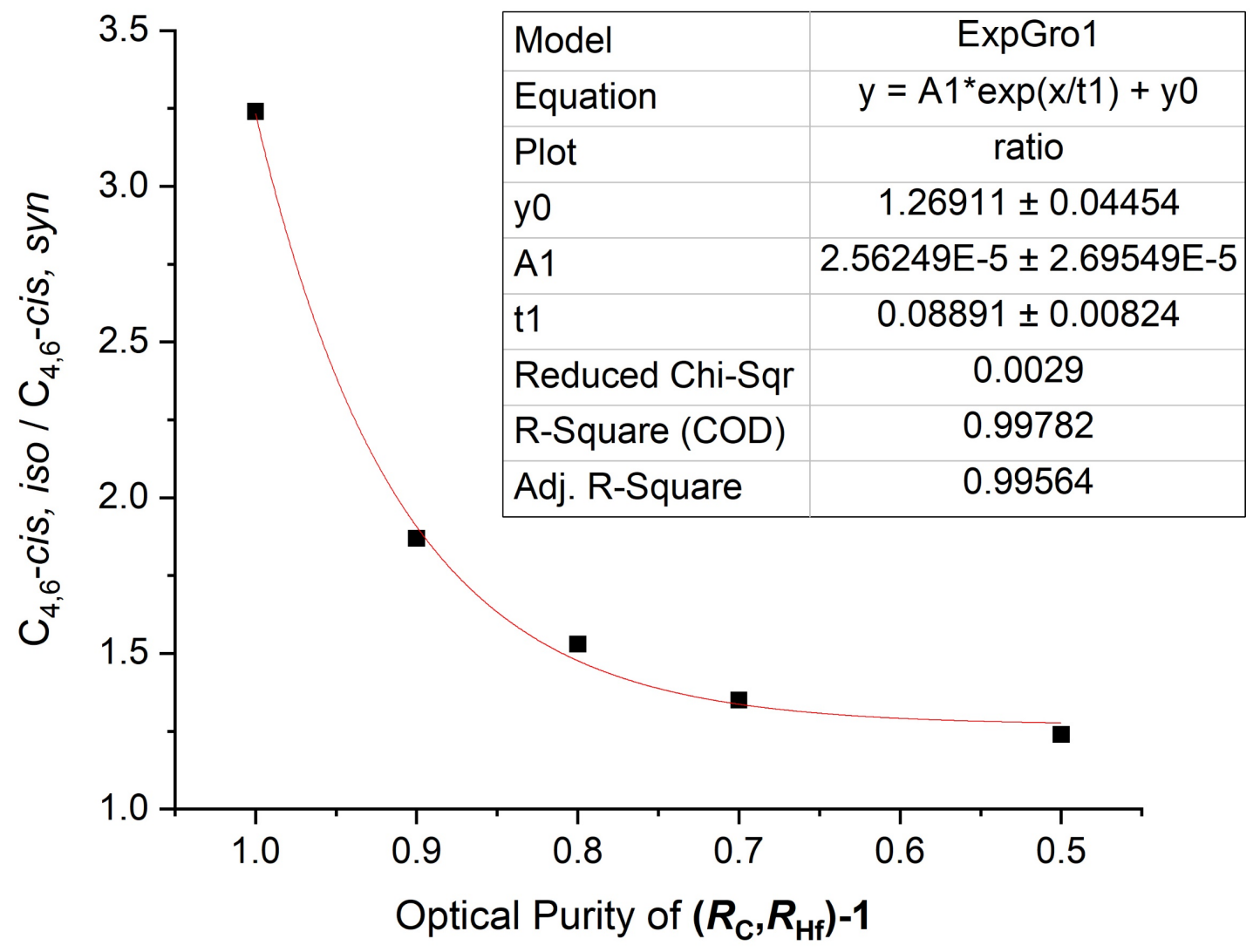




\section{References}

1. Wallace, M. A.; Zavalij, P. Y.; Sita, L. R. Enantioselective Living Coordinative Chain Transfer Polymerization: Production of Optically Active End-Group-Functionalized (+)or (-)-Poly(methylene-1,3-cyclopentane) via a Homochiral C1-Symmetric Caproamidinate Hafnium Initiator. ACS Catal. 2020, 10, 8496-8502.

2. Crawford, K. E.; Sita, L. R. Stereoengineering of Poly(1,3-methylenecyclohexane) via Two-State Living Coordination Polymerization of 1,6-Heptadiene. J. Am. Chem. Soc. 2013, 135, 8778-8781.

3. Zhang, W.; Wei, J.; Sita, L. R. Living Coordinative Chain-Transfer Polymerization and Copolymerization of ethene, $\alpha$-Olefins, and $\alpha, \omega$-Nonconjugated Dienes using Dialkylzinc as "Surrogate" Chain-Growth Sites. Macromolecules. 2008, 41, 78297833.

4. Coates, G. W.; Waymouth, R. M. Enantioselective Cyclopolymerization of 1,5Hexadiene Catalyzed by Chiral Zirconocenes: A Novel Strategy for the Synthesis of Optically Active Polymers with Chirality in the Main Chain. J. Am. Chem. Soc. 1993, 115, 91-98 